\title{
Canonical quantization on the half-line and in an interval based upon an alternative concept for the momentum in a space with boundaries
}

\author{
M. H. Al-Hashimi $\odot$ and U.-J. Wiese $\odot$ \\ Albert Einstein Center, Institute for Theoretical Physics, University of Bern, 3012 Bern, Switzerland
}

(Received 3 March 2021; accepted 1 July 2021; published 21 July 2021)

\begin{abstract}
For a particle moving on a half-line or in an interval the operator $\hat{p}=-i \partial_{x}$ is not self-adjoint and thus does not qualify as the physical momentum. Consequently canonical quantization based on $\hat{p}$ fails. Based upon an alternative concept for a self-adjoint momentum operator $\hat{p}_{R}$, we show that canonical quantization can indeed be implemented on the half-line and on an interval. Both the Hamiltonian $\hat{H}$ and the momentum operator $\hat{p}_{R}$ are endowed with self-adjoint extension parameters that characterize the corresponding domains $D(\hat{H})$ and $D\left(\hat{p}_{R}\right)$ in the Hilbert space. When one replaces Poisson brackets by commutators, one obtains meaningful results only if the corresponding operator domains are properly taken into account. The alternative concept for the momentum is used to describe the results of momentum measurements of a quantum mechanical particle that is reflected at impenetrable boundaries, either at the end of the half-line or at the two ends of an interval.
\end{abstract}

DOI: 10.1103/PhysRevResearch.3.033079

\section{INTRODUCTION}

Momentum is one of the most fundamental physical quantities. The momentum operator generates infinitesimal translations in infinite space. When the Hamiltonian is translation invariant, momentum is conserved. Coordinates and their canonically conjugate momenta play a central role in Hamiltonian dynamics, which turns into quantum mechanics upon canonical quantization.

Several important physical systems are confined inside a limited region of space with sharp boundaries. These include, for example, ultracold atoms in an optical box trap [1] of a few tens of micrometers in size, electrons in a quantum dot at the nanometer scale [2], the "femto-universe" of the phenomenological MIT bag model [3-5] for confined quarks and gluons, domain wall fermions [6,7], or intervals of extra-dimensional space at the Planck scale. In the idealized mathematical description of such systems, space is endowed with sharp impenetrable boundaries. While for most systems this is just a mathematical convenience that allows one to exclude regions of very high potential energy, it is conceivable that extra-dimensional space literally ends at a boundary. In spaces with boundaries, translation invariance is explicitly broken, not just because the Hamiltonian includes symmetrybreaking terms, but because space itself ends (at least in the idealized mathematical description). As a consequence, the usual quantum mechanical momentum operator $\hat{p}=-i \partial_{x}$ is no longer self-adjoint, and thus no longer represents the physical momentum. From this it has been concluded that,

Published by the American Physical Society under the terms of the Creative Commons Attribution 4.0 International license. Further distribution of this work must maintain attribution to the author(s) and the published article's title, journal citation, and DOI. in a finite volume, momentum is no longer a measurable physical observable [8]. As another consequence of the nonself-adjointness of $\hat{p}$, canonical quantization (which is based upon $\hat{p}$ ) fails for confined systems with sharp boundaries.

Recently, we have introduced an alternative concept for the momentum of a quantum mechanical particle in a box [9], which gives rise to a physically and mathematically satisfactory self-adjoint momentum operator. This concept naturally extends to the half-line as well as to higher dimensions. Intervals and half-lines are the basic building blocks of higher-dimensional regions of space with sharp boundaries. The present investigations hence form the basis for future applications to physical systems in higher dimensions. The sharp boundaries of a confined system give rise to a high degree of ultraviolet sensitivity. At low energies, this reflects itself in the values of self-adjoint extension parameters of the Hamiltonian that characterize the boundary conditions of the wave function.

In order to take the ultraviolet sensitivity into account properly, in our construction of a self-adjoint momentum operator in [9] we started out from an ultraviolet lattice regularization. While this is familiar in nonperturbative quantum field theory, in this case it is beneficial even in quantum mechanics. On the lattice, one naturally distinguishes forward and backward derivatives, neither of them being Hermitian. The Hermitian lattice momentum operator is given by a symmetrized forward-backward derivative, which manifests itself as a finite difference that extends over two lattice spacings. This naturally leads to the distinction of even and odd lattice points. A careful analysis of the problem reveals that the construction of a self-adjoint momentum operator in the continuum limit requires the doubling of the standard Hilbert space, in order to maintain a remnant of the crucial distinction between even and odd lattice points even in the continuum [9]. In fact, some aspects of the problem are reminiscent of the lattice fermion doubling problem [10-12], which arises because the Dirac 
operator contains first-order derivatives. The resulting insight of [9] is that the construction of a self-adjoint momentum operator requires a refined concept, not only of Hilbert space, but even of space itself. This may not be too surprising, because a space that ends abruptly supports other momentum modes than infinite space. Still, as we will see, the finite-energy sector, which is defined by the Hamiltonian, resides in a region of Hilbert space that is completely equivalent to the standard quantum mechanical treatment.

An extension of the Hilbert space also plays an important role in quantum measurements related to a positive operatorvalued measure (POVM) [13-19], which are based on Kraus operators describing quantum jumps. Measurement processes using a POVM provide a generalization of von Neumann's standard projective measurements. They play an important role for controlling quantum systems and processing quantum information. In POVM-based measurements, the quantum system to be investigated is first coupled to another quantum system that acts as an ancilla. Then a standard projective measurement is performed on the ancilla, which indirectly affects the quantum system under study [20]. That a given POVM can be realized by an appropriate extension of the Hilbert space is related to Neumark's theorem [18,21-24]. In this way measurements on confined systems have been described by POVMs [25] and an optimal POVM for a particle on a half-line has been considered in [26]. It should be pointed out that the POVM measurement is not based on a self-adjoint momentum operator of the quantum particle itself, but rather of the particle coupled to its ancilla. Our construction, on the other hand, provides a self-adjoint momentum operator for the particle alone (without invoking any ancilla). In our case, the doubling of the Hilbert space results from the necessity to include states whose energy is ultraviolet-sensitive, but which, due to the existence of sharp boundaries, still contribute to the momentum eigenstates. In other words, the Hilbert space that contains the finite-energy eigenstates is too small to contain also the momentum eigenstates of a quantum particle in a space with sharp boundaries.

The time evolution of a quantum system is driven by its Hamiltonian, which is described by a self-adjoint operator acting in an appropriate Hilbert space. In nonrelativistic 1d quantum mechanics, the single-particle Hamiltonian $\hat{H}=$ $-\frac{1}{2 m} \partial_{x}^{2}+V(x)$ (in units where $\hbar=1$ ) contains the differential operator $\partial_{x}^{2}$, which is supposed to act on square-integrable wave functions. Since not all square-integrable functions are differentiable, the Hamiltonian (as well as other physical operators) acts only in a restricted domain $D(\hat{H})$ of the Hilbert space. For a differential operator the domain is characterized by the square-integrability of the corresponding derivatives of the wave function. In an infinite-dimensional Hilbert space, there are subtle differences between Hermiticity and selfadjointness, which were first understood by von Neumann [27]. Hermiticity means that an operator $\hat{A}$ and its adjoint $\hat{A}^{\dagger}$ act in the same way. Self-adjointness requires, in addition, that the corresponding domains $D(\hat{A})=D\left(\hat{A}^{\dagger}\right)$ coincide [28-30]. In order to qualify as a physical observable, an operator must be self-adjoint. This is because only self-adjointness, and not Hermiticity alone, guarantees a spectrum of real eigenvalues with a corresponding complete set of orthonormal eigenfunctions. During its time evolution the wave function of a particle with finite energy only explores the domain $D(\hat{H})$, and never reaches other corners in Hilbert space.

However, when the unitary time evolution driven by the Hamiltonian is interrupted by an (idealized) projective measurement, the momentary wave function is projected onto an eigenstate of the operator $\hat{A}$ that describes the measured observable. It is possible that the domains of the Hamiltonian $D(\hat{H})$ and of the observable $D(\hat{A})$ do not coincide. This is no problem, because a self-adjoint operator has a complete set of eigenfunctions. As a consequence, every wave function [even outside of $D(\hat{A})$ ] can be represented arbitrarily well by a superposition of eigenstates of $\hat{A}$. In this way, one can determine the probabilities to measure the various possible eigenvalues of the observable $\hat{A}$. After such a projective measurement, the wave function is inside $D(\hat{A})$ but not necessarily any longer inside $D(\hat{H})$. How can the unitary time evolution proceed after such a measurement? Again, since the Hamiltonian is self-adjoint, any state [even outside $D(\hat{H})$ ] can be approximated arbitrarily well by a superposition of eigenstates of $\hat{H}$, and the time evolution proceeds accordingly. However, the energy expectation value after a measurement that leads out of $D(\hat{H})$ is usually infinite. Hence, idealized measurements can transfer an infinite amount of energy to the particle under investigation. Of course, any practical measurement only consumes a finite amount of energy, and is, in any case, not completely realistically described by an idealized projective measurement.

Momentum measurements on confined particles fall in this category. For example, a particle of finite energy that moves along the entire real axis in a potential that diverges at spatial infinity, $V( \pm \infty) \rightarrow \infty$, has a square-integrable wave function that vanishes at infinity and belongs to the Hilbert space $L^{2}(\mathbb{R})$ of square-integrable wave functions over the entire real axis. When the momentum of the particle is measured and one obtains the value $k$, its wave function collapses onto the plane wave momentum eigenstate $\langle x \mid k\rangle=\exp (i k x)$, which has $\langle k|V| k\rangle=\infty$. It should be pointed out that this state does not belong to the Hilbert space, because it is not square-integrable. As a result, a standard instantaneous projective momentum measurement, which results in an exact momentum eigenstate after the measurement, indeed transfers an infinite amount of energy to a confined particle. This is a result of von Neumann's concept of idealized projective measurements, which does not do justice to all aspects of actual measurements performed inside a laboratory. In particular, those have finite resolution, they take a finite amount of time, and they transfer only a finite amount of energy. While being aware of these facts, in this work we follow the completely standard practice of using von Neumann's concept of idealized projective measurements.

The situation becomes more subtle in the presence of impenetrable sharp boundaries (which, of course, again are mathematical idealizations) [8,31-35]. For example, for a particle that is strictly confined to the positive real axis (the half-line) the operator $\hat{p}=-i \partial_{x}$ is not self-adjoint, and hence it has until now been unknown how to properly define the corresponding momentum operator. As a consequence, one has replaced the momentum operator by the dilation operator, thus moving from canonical to affine quantization [36-40]. The main purpose of this paper is to provide an 
appropriate construction of a self-adjoint momentum operator that is satisfactory both from a physical and from a mathematical point of view, and to show that canonical quantization is, in fact, applicable to the half-line as well as to an interval. As a result, we will be able to describe momentum measurements performed on a quantum mechanical particle that is strictly limited to the positive real axis or to an interval, even after a momentum measurement. Such a particle is bound to or reflected at impenetrable boundaries, either at the origin or at the two ends of the interval.

It should be pointed out that our construction of a selfadjoint momentum operator assumes that some physically meaningful ultraviolet cutoff (like a crystal lattice in a quantum dot) actually exists, and that the quantum mechanical description in the continuum is an effective low-energy description that is valid only below that ultraviolet cutoff. If one assumes that the quantum mechanics formulated in the continuum is a "theory of everything," in other words that no physical ultraviolet cutoff exists at short distances, there would be no basis for extending the Hilbert space. In that case, momentum would indeed not be a meaningful concept for motion on the half-line, and one would be restricted to affine quantization. In experimental situations involving, for example, quantum dots, there is always a physical ultraviolet cutoff at which the effective low-energy quantum mechanical description in the continuum breaks down, such that our momentum concept is indeed applicable. Whether the Planck length leads to a physical ultraviolet cutoff in extradimensional spaces with boundaries is, of course, a matter of speculation.

The paper is written also with some pedagogical intentions in mind. Therefore we do not assume that the reader is familiar with the concept of operator domains, which is crucial for the distinction between Hermiticity and self-adjointness. Unfortunately, in the education of the typical theoretical physicist these issues often do not play a prominent role. The experts will hopefully not be offended that we elaborate on some issues that are well known to them. We also like to point out that the notion of canonical quantization is not as uniquely defined as one might think. Definitely, it describes quantization in an equal-time Hamiltonian formulation, rather than, for example, on a light cone or some other hypersurface. Here we use a more narrow definition of canonical quantization, which is based upon the canonical commutation relations between coordinates and conjugate momenta (or closely related variants thereof). This definition distinguishes canonical quantization from affine quantization, which also operates in an equal-time Hamiltonian framework, but replaces the momentum by the dilation operator. The main result of our work is the construction of an appropriate self-adjoint momentum operator, which forms the basis for successfully applying canonical quantization to the half-line and to an interval, for which it was thought to be inapplicable. Still, applying canonical quantization (defined in this way) to the half-line or an interval is less straightforward than for the entire real axis, because some subtleties related to operator domains are crucial. Although this is well known to the experts, we will discuss explicitly how canonical quantization should be applied in such cases.

The rest of the paper is organized as follows. In Sec. II, we address the non-self-adjointness of the standard momen- tum operator $\hat{p}=-i \partial_{x}$ on the half-line $\mathbb{R}_{\geqslant 0}$, we construct the self-adjoint extensions of the Hamiltonian, and we address canonical quantization and standard momentum measurements, as well as affine quantization. Based upon our alternative concept, in Sec. III we construct a self-adjoint momentum operator on the half-line, embed the Hamiltonian in the resulting mathematical framework, and discuss the corresponding momentum measurements. We then consider the resulting canonical quantization on the half-line including the classical limit. Section IV addresses canonical quantization on an interval and relates the results to the situation on a circle. Finally, we end with some conclusions. Ultraviolet lattice aspects of our momentum concept are summarized in an Appendix.

\section{FROM CANONICAL QUANTIZATION ON THE ENTIRE REAL AXIS TO AFFINE QUANTIZATION ON THE HALF-LINE}

In this section we address the non-self-adjointness of the standard momentum operator on the half-line and its consequences for canonical and affine quantization.

\section{A. Non-self-adjointness of $-i \partial_{x}$ on $\mathbb{R}_{\geqslant 0}$}

Let us consider the standard momentum operator $\hat{p}=-i \partial_{x}$ on the half-line $\mathbb{R}_{\geqslant 0}$. Using partial integration one obtains

$$
\left\langle\hat{p}^{\dagger} \chi \mid \Psi\right\rangle=\langle\chi \mid \hat{p} \Psi\rangle=\langle\hat{p} \chi \mid \Psi\rangle-i \chi(0)^{*} \Psi(0) .
$$

Hermiticity requires that $\chi(0)^{*} \Psi(0)=0$. This requirement can be satisfied if one restricts the domain $D(\hat{p})$ to those wave functions whose derivative is square-integrable and that obey $\Psi(0)=0$. However, then $\chi(0)$ remains unrestricted and can still assume arbitrary values. Consequently, the domain of $\hat{p}^{\dagger}$, which acts on $\chi$, remains unrestricted, and $D\left(\hat{p}^{\dagger}\right) \supset D(\hat{p})$. When $\Psi(0)=0, \hat{p}$ and $\hat{p}^{\dagger}$ act in the same way and hence $\hat{p}=-i \partial_{x}$ is indeed Hermitian. However, since $D\left(\hat{p}^{\dagger}\right) \neq D(\hat{p})$, it is not self-adjoint. In fact, it is impossible to extend $\hat{p}$ to a self-adjoint operator on the half-line. Consequently, $\hat{p}=-i \partial_{x}$ does not describe the physical momentum of a quantum mechanical particle that moves along the positive real axis. As a result, it has been concluded that, in this case, momentum is no longer an observable physical quantity [8]. We will reach a different conclusion, namely that not $\hat{p}=-i \partial_{x}$ (which is not self-adjoint) but another operator, $\hat{p}_{R}=-i \sigma_{1} \partial_{x}$, which is self-adjoint in the Hilbert space $L^{2}\left(\mathbb{R}_{\geqslant 0}\right) \times \mathbb{C}^{2}$ of the doubly covered positive real axis, describes the physical momentum of a particle on the half-line. In fact, the appropriate momentum operator $\hat{p}_{R}+i \hat{p}_{I}$ has a Hermitian component $\hat{p}_{R}$ as well as an anti-Hermitian component $i \hat{p}_{I}$, with both $\hat{p}_{R}$ and $\hat{p}_{I}$ being self-adjoint.

\section{B. Self-adjoint extension of $\hat{\boldsymbol{H}}$ on $\mathbb{R}_{\geqslant 0}$}

When restricted to the half-line, the self-adjointness of $\hat{H}=-\frac{1}{2 m} \partial_{x}^{2}+V(x)$, with $V(x)$ being nonsingular, requires the following adaptations. First of all, by performing two 
partial integrations one obtains

$$
\begin{aligned}
\left\langle\hat{H}^{\dagger} \chi \mid \Psi\right\rangle & =\langle\chi \mid \hat{H} \Psi\rangle \\
& =\langle\hat{H} \chi \mid \Psi\rangle-\frac{1}{2 m}\left[\partial_{x} \chi(0)^{*} \Psi(0)-\chi(0)^{*} \partial_{x} \Psi(0)\right] .
\end{aligned}
$$

Hermiticity hence requires the term in square brackets to vanish. The most general boundary condition that is consistent with the linearity of quantum mechanics is the Robin boundary condition

$$
\gamma \Psi(0)-\partial_{x} \Psi(0)=0 .
$$

Dirichlet boundary conditions, $\Psi(0)=0$, result from $\gamma \rightarrow$ $\infty$, while Neumann boundary conditions, $\partial_{x} \Psi(0)=0$, correspond to $\gamma=0$. Since (for finite $\gamma$ ) $\Psi(0)$ itself can still take arbitrary values, inserting Eq. (3) in the square bracket in Eq. (2), the Hermiticity condition turns into

$$
\left[\partial_{x} \chi(0)^{*}-\gamma \chi(0)^{*}\right] \Psi(0)=0 \Rightarrow \gamma^{*} \chi(0)-\partial_{x} \chi(0)=0 \text {. }
$$

This relation characterizes the domain $D\left(\hat{H}^{\dagger}\right)$ of $\hat{H}^{\dagger}$ (which acts on $\chi)$. The Hamiltonian is self-adjoint only if the two domains coincide, $D\left(\hat{H}^{\dagger}\right)=D(\hat{H})$, i.e., if $\gamma^{*}=\gamma \in \mathbb{R}$. In this way, we obtain a 1-parameter family of self-adjoint extensions of $\hat{H}$.

Self-adjointness is not just a mathematical requirement; it also has most important physical consequences. In particular, for $\gamma \in \mathbb{R}$ the boundary condition of Eq. (3) ensures that the probability current density,

$$
j(x)=\frac{1}{2 m i}\left[\Psi(x)^{*} \partial_{x} \Psi(x)-\partial_{x} \Psi(x)^{*} \Psi(x)\right],
$$

vanishes at the boundary, i.e., $j(0)=0$, and hence does not flow into the forbidden region on the negative real axis. This ensures unitarity, i.e., probability conservation, for the particle moving along the half-line.

In the absence of a potential $[V(x)=0]$ it is easy to construct the energy eigenstates. First of all, there are stationary scattering states of positive energy $E=\frac{p^{2}}{2 m}$ :

$$
\psi_{E}(x)=\exp (-i p x)+R(p) \exp (i p x), R(p)=\frac{i p+\gamma}{i p-\gamma} .
$$

It is sufficient to limit oneself to $p \geqslant 0$, because (together with a bound state for $\gamma<0$ ) these states alone form a complete orthonormalized set with

$$
\left\langle\psi_{E^{\prime}} \mid \psi_{E}\right\rangle=2 \pi \delta\left(p-p^{\prime}\right),
$$

where $E^{\prime}=\frac{p^{\prime 2}}{2 m}, p^{\prime}>0$. In particular, since $R(-p)=R(p)^{*}=$ $R(p)^{-1}$, the states with opposite values of $p$ are simply given by

$$
\exp (i p x)+R(-p) \exp (-i p x)=R(-p) \psi_{E}(x) .
$$

In addition, for $\gamma<0$, there is a bound state of negative energy

$$
\psi_{b}(x)=\sqrt{-2 \gamma} \exp (\gamma x), \quad E_{b}=-\frac{\gamma^{2}}{2 m} .
$$

Interestingly, a perfectly reflecting impenetrable barrier can still support bound states, and the Hamiltonian $\hat{H}=-\frac{1}{2 m} \partial_{x}^{2}$

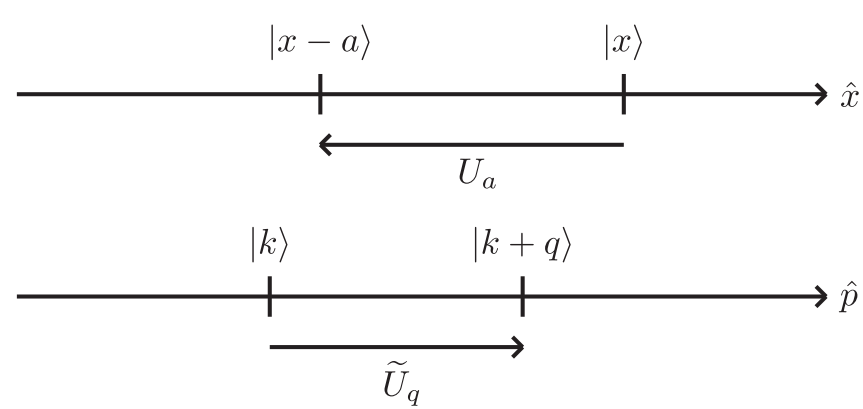

FIG. 1. Action of the translation operators $U_{a}=\exp (i \hat{p} a)$ and $\widetilde{U}_{q}=\exp (i q \hat{x})$ on the position and momentum eigenstates for the entire real axis $\mathbb{R}$.

(endowed with a negative self-adjoint extension parameter $\gamma<0$ ) indeed has an eigenstate of negative energy. It is easy to convince oneself that the bound state is orthogonal to the scattering states, i.e., $\left\langle\psi_{E} \mid \psi_{b}\right\rangle=0$.

\section{Canonical quantization on $\mathbb{R}$}

Canonical quantization is based upon the canonical commutation relation $[\hat{x}, \hat{p}]=i$. This relation applies to unrestricted linear motion, because in the Hilbert space $L^{2}(\mathbb{R})$ the momentum operator $\hat{p}=-i \partial_{x}$ is indeed self-adjoint. The operator $\hat{p}$ then generates infinitesimal translations in coordinate space. The unitary operator that translates a wave function by a distance $-a$ acts as

$$
U_{a} \Psi(x)=\exp (i \hat{p} a) \Psi(x)=\exp \left(a \partial_{x}\right) \Psi(x)=\Psi(x+a) .
$$

By a Fourier transformation, we obtain the momentum space wave function

$$
\begin{aligned}
\widetilde{\Psi}(p) & =\langle p \mid \Psi\rangle=\int_{-\infty}^{\infty} d x\langle p \mid x\rangle\langle x \mid \Psi\rangle \\
& =\int_{-\infty}^{\infty} d x \exp (-i p x) \Psi(x) .
\end{aligned}
$$

The position operator $\hat{x}=i \partial_{p}$ generates infinitesimal translations in momentum space. The unitary operator $\widetilde{U}_{q}=$ $\exp (i q \hat{x})$, which translates a momentum space wave function by $q$, acts as

$$
\widetilde{U}_{q} \widetilde{\Psi}(p)=\exp \left(-q \partial_{p}\right) \widetilde{\Psi}(p)=\widetilde{\Psi}(p-q) .
$$

For momentum eigenstates $\langle x \mid k\rangle=\exp (i k x)$ [which are orthonormalized to $\left.\left\langle k^{\prime} \mid k\right\rangle=2 \pi \delta\left(k-k^{\prime}\right)\right]$ we have

$$
\langle x \mid k+q\rangle=\exp (i q x)\langle x \mid k\rangle=\widetilde{U}_{q}\langle x \mid k\rangle .
$$

We also find

$$
\begin{aligned}
& U_{a} \widetilde{U}_{q} \Psi(x)=U_{a} \exp (i q x) \Psi(x)=\exp [i q(x+a)] \Psi(x+a), \\
& \widetilde{U}_{q} U_{a} \Psi(x)=\exp (\operatorname{iq} x) \Psi(x+a) .
\end{aligned}
$$

Hence, as a counterpart to the Heisenberg algebra $[\hat{x}, \hat{p}]=i$, one obtains the Weyl group relation

$$
U_{a} \widetilde{U}_{q}=\exp (\text { iqa }) \widetilde{U}_{q} U_{a} .
$$

The action of the operators $U_{a}$ and $\widetilde{U}_{q}$ is illustrated in Fig. 1. 


\section{Standard momentum measurements}

Since the standard momentum operator $\hat{p}=-i \partial_{x}$ is selfadjoint only over the entire real axis but not over the half-line, applying the standard concept of a momentum measurement requires extending the Hilbert space from $L^{2}\left(\mathbb{R}_{\geqslant 0}\right)$ to $L^{2}(\mathbb{R})$ [41]. This can be achieved by assigning a finite constant potential $V(x<0)=V_{0}$ to the negative real axis and finally sending $V_{0} \rightarrow \infty$ [42]. A momentum measurement then projects the wave function (which is exponentially suppressed on the negative real axis) on a plane wave $\langle x \mid k\rangle=\exp (i k x)$, which is unsuppressed for $x<0$. Such a momentum measurement catapults the particle out of the energetically allowed region and transfers an infinite amount of energy to the particle in the limit $V_{0} \rightarrow \infty$. Such a measurement can be realized, for example, in an optical box trap, if the ultracold atoms are released from the trap immediately before the momentum measurement.

Let us first consider the bound state $\left|\psi_{b}\right\rangle$ for $\gamma<0$. It may not be obvious that the bound state can arise in a context in which $V_{0}$ approaches infinity from finite values. Indeed, with a standard finite square-well potential, this would not be the case. However, as shown explicitly in [35], by taking an appropriate limit of a finite piecewise constant potential, one can realize any desirable value of $\gamma$. The overlap of the bound state with the eigenstates $|k\rangle$ determines the probability density to obtain the value $k$ in a measurement of the standard momentum operator

$$
\frac{1}{2 \pi}\left|\left\langle k \mid \psi_{b}\right\rangle\right|^{2}=-\frac{1}{\pi} \frac{\gamma}{\gamma^{2}+k^{2}} .
$$

As expected, the resulting momentum expectation value vanishes. The momentum uncertainty diverges because

$$
\frac{1}{2 \pi} \int_{-\infty}^{\infty} d k k^{2}\left|\left\langle k \mid \psi_{b}\right\rangle\right|^{2}=-\frac{1}{\pi} \int_{-\infty}^{\infty} d k \frac{\gamma k^{2}}{\gamma^{2}+k^{2}}=\infty .
$$

In the limit $\gamma \rightarrow 0^{-}$the bound state becomes an unbound zero-energy scattering state, and the probability density to measure the momentum $k$ turns into

$$
-\lim _{\gamma \rightarrow 0^{-}} \frac{1}{\pi} \frac{\gamma}{\gamma^{2}+k^{2}}=\delta(k) .
$$

This seems to suggest that, in this limit, the value $k=0$ is measured with certainty, in contradiction to the divergent uncertainty that we found for $\gamma<0$. This shows that the limit $\gamma \rightarrow 0^{-}$is not approached uniformly. In any case, the state $\psi_{b}(x)=A \theta(x)$, which in the limit $\gamma \rightarrow 0^{-}$becomes proportional to the step function $\theta(x)$, is not identical with the $k=0$ momentum eigenstate, which is constant over the entire real axis. This property results from the fact that $\left|\psi_{b}\right\rangle$ belongs to the Hilbert space $L^{2}\left(\mathbb{R}_{\geqslant 0}\right)$ while $|k\rangle$ resides in an extension of $L^{2}(\mathbb{R})$.

Next, we consider standard momentum measurements performed on the positive energy scattering state $\left|\psi_{E}\right\rangle$ with $E=$ $\frac{p^{2}}{2 m}, p \geqslant 0$. It is straightforward to obtain

$$
\left\langle k \mid \psi_{E}\right\rangle=-i \lim _{\epsilon \rightarrow 0^{+}}\left(\frac{1}{k-i \epsilon+p}+R(p) \frac{1}{k-i \epsilon-p}\right) .
$$

Using the residue theorem one then confirms that

$$
\left\langle\psi_{E^{\prime}} \mid \psi_{E}\right\rangle=\frac{1}{2 \pi} \int_{-\infty}^{\infty} d k\left\langle\psi_{E^{\prime}} \mid k\right\rangle\left\langle k \mid \psi_{E}\right\rangle=2 \pi \delta\left(p-p^{\prime}\right) .
$$

In this case, one might expect that the only possible measurement results for the standard momentum are $k=p$ and $k=-p$, each with probability $\frac{1}{2}$. However, as we will see at the end of the paper, these probabilities are only $\frac{1}{4}$. Due to the two different Hilbert spaces $L^{2}\left(\mathbb{R}_{\geqslant 0}\right)$ and $L^{2}(\mathbb{R}), \psi_{E}(x)$, which vanishes for $x<0$, is not a linear combination of $\langle x \mid-p\rangle=\exp (-i p x)$ and $R(p)\langle x \mid p\rangle=R(p) \exp (i p x)$ along the entire real axis.

We may conclude that it is possible to enforce the standard concept of momentum for a particle on the half-line, however, at the price of putting the particle also onto the negative real axis as a result of the measurement. We will soon present an alternative concept for a self-adjoint momentum operator, which, on the half-line, leads to the same probability distribution for the measurement results, but strictly confines the particle to the positive real axis also after a measurement. In a finite interval, on the other hand, the momentum values associated with the alternative concept are discrete and thus differ qualitatively from the continuous values of the standard momentum.

\section{E. Affine quantization on $\mathbb{R}_{\geqslant 0}$}

Since on the half-line there is no translation invariance, the operator $\hat{p}=-i \partial_{x}$ is not self-adjoint. As a result, the standard commutation relation $[\hat{x}, \hat{p}]=i$ that underlies canonical quantization has only a formal status and is no longer physically meaningful. Consequently, in this case canonical quantization based upon $\hat{p}$ fails. For this reason, on the half-line affine quantization has played an important role [36-40]. The momentum operator $\hat{p}$ is then replaced by the generator $\hat{d}$ of infinitesimal dilations. The unitary operator that dilates a wave function by a scale factor $1 / s \in \mathbb{R}_{>0}$ acts as

$$
U_{s} \Psi(x)=\exp (i \hat{d} \ln s) \Psi(x)=\sqrt{s} \Psi(s x) .
$$

Let us consider an infinitesimal dilation $s=1+\epsilon$ with small $\epsilon$. We then obtain

$$
\begin{aligned}
U_{s} \Psi(x) & =\sqrt{1+\epsilon} \Psi(x+\epsilon x) \\
& \approx\left(1+\frac{\epsilon}{2}\right)\left[\Psi(x)+\epsilon x \partial_{x} \Psi(x)\right] \\
& \approx(1+i \epsilon \hat{d}) \Psi(x) \Rightarrow \\
\hat{d} \Psi(x) & =-i\left(\frac{1}{2}+x \partial_{x}\right) \Psi(x) \Rightarrow \\
\hat{d} & =-i\left(\frac{1}{2}+x \partial_{x}\right)=-\frac{i}{2}\left(\partial_{x} x+x \partial_{x}\right) .
\end{aligned}
$$

The commutation relation that replaces $[\hat{x}, \hat{p}]=i$ in affine quantization is

$$
[\hat{x}, \hat{d}]=\left[\hat{x},-i x \partial_{x}\right]=i \hat{x} .
$$


By performing a partial integration we obtain

$$
\begin{aligned}
\left\langle\hat{d}^{\dagger} \chi \mid \Psi\right\rangle & =\langle\chi \mid \hat{d} \Psi\rangle \\
& =\int_{0}^{\infty} d x \chi(x)^{*}\left[-i\left(\frac{1}{2}+x \partial_{x}\right) \Psi(x)\right] \\
& =\int_{0}^{\infty} d x\left[-i\left(\frac{1}{2}+x \partial_{x}\right) \chi(x)\right]^{*} \Psi(x) \\
& =\langle\hat{d} \chi \mid \Psi\rangle .
\end{aligned}
$$

As long as $x \chi(x)^{*} \Psi(x)$ vanishes at the boundary $x=0, \hat{d}$ is Hermitian. Since square-integrable wave functions are less singular than $1 / \sqrt{x}$ near the origin, this condition is satisfied without further domain restrictions. As a result, $D(\hat{d})=$ $D\left(\hat{d}^{\dagger}\right)$ and $\hat{d}$ is indeed self-adjoint.

The eigenfunctions of $\hat{d}$ obey

$$
\begin{aligned}
& \hat{d}|\varkappa\rangle=-i\left(\frac{1}{2}+x \partial_{x}\right)|\varkappa\rangle=\varkappa|\varkappa\rangle \Rightarrow \\
& \langle x \mid \varkappa\rangle=\frac{1}{\sqrt{l}}\left(\frac{x}{l}\right)^{i \varkappa-1 / 2} .
\end{aligned}
$$

Here $l$ is an arbitrarily chosen fixed length scale. Similarly to the momentum eigenstates $|k\rangle$ over $\mathbb{R}$, the eigenstates $|\varkappa\rangle$ of $\hat{d}$ are not normalizable in the usual sense. The analog of Fourier transformation for canonical quantization is a Mellintype transformation [36] for affine quantization

$$
\begin{aligned}
\tilde{\Psi}(\varkappa) & =\langle\varkappa \mid \Psi\rangle=\int_{0}^{\infty} d x\langle\varkappa \mid x\rangle\langle x \mid \Psi\rangle \\
& =\int_{0}^{\infty} d x \frac{1}{\sqrt{l}}\left(\frac{x}{l}\right)^{-i \varkappa-1 / 2} \Psi(x) .
\end{aligned}
$$

Let us also introduce the unitary operator $\widetilde{U}_{\delta}=\exp (i \delta \hat{x})$ which leads to

$$
\begin{aligned}
U_{s} \widetilde{U}_{\delta} \Psi(x) & =U_{s} \exp (i \delta x) \Psi(x)=\sqrt{s} \exp (i \delta s x) \Psi(s x), \\
\widetilde{U}_{\delta s} U_{s} \Psi(x) & =\exp (i \delta s x) \sqrt{s} \Psi(s x) .
\end{aligned}
$$

Hence, in analogy to the Weyl group for canonical quantization, for affine quantization one obtains the affine group relation

$$
U_{s} \widetilde{U}_{\delta}=\tilde{U}_{\delta s} U_{s}
$$

\section{FROM THE ALTERNATIVE CONCEPT FOR THE MOMENTUM OPERATOR TO CANONICAL QUANTIZATION ON THE HALF-LINE}

In this section, we introduce an alternative concept for the momentum operator in a space with sharp boundaries, which allows us to apply canonical quantization to the half-line.

\section{A. A self-adjoint momentum operator on the half-line}

Recently we have developed an alternative concept for the momentum of a quantum mechanical particle in a 1-d box $\left[-\frac{L}{2}, \frac{L}{2}\right]$ [9], which readily extends to the half-line as well as to higher-dimensional spaces with sharp boundaries. For the convenience of the reader, we summarize the most important aspects of this construction in the Appendix. The construction results from the continuum limit of a system that is regularized on a spatial lattice. On a lattice, the derivative that enters the momentum operator is replaced by a nearestneighbor finite difference. One must distinguish forward and backward derivatives, neither of them being Hermitian. Only the symmetrized forward-backward derivative, which corresponds to a next-to-nearest neighbor finite difference that extends over two lattice spacings, results in a Hermitian momentum operator. The lattice is naturally divided into two sublattices, one with even and one with odd lattice sites. The symmetrized forward-backward derivative associated with an even site then results from the values of the wave function at the two neighboring odd sites. In the continuum limit, the sublattice structure naturally leads to a two-component wave function, on which the momentum operator acts as a $2 \times 2$ matrix

$$
\hat{p}_{R}=-i\left(\begin{array}{cc}
0 & \partial_{x} \\
\partial_{x} & 0
\end{array}\right)=-i \sigma_{1} \partial_{x}, \Psi(x)=\left(\begin{array}{l}
\Psi_{e}(x) \\
\Psi_{o}(x)
\end{array}\right) .
$$

As a result, the problem is elevated to the Hilbert space $L^{2}\left(\mathbb{R}_{\geqslant 0}\right) \times \mathbb{C}^{2}$ of square-integrable functions on the double cover of the half-line. This is the crucial insight that leads to the construction of a self-adjoint momentum operator.

The full momentum operator $\hat{p}_{R}+i \hat{p}_{I}$ has both a Hermitian component $\hat{p}_{R}$ (which can be extended to a self-adjoint operator) and an anti-Hermitian component $i \hat{p}_{I}$ with

$$
\hat{p}_{I}=\lim _{\epsilon \rightarrow 0^{+}}\left(\begin{array}{cc}
\delta(x-\epsilon) & 0 \\
0 & 0
\end{array}\right) .
$$

The operator $\hat{p}_{I}$ is self-adjoint and diagonal in the position basis.

Let us first investigate the Hermiticity of $\hat{p}_{R}$. By partial integration we obtain

$$
\begin{aligned}
\left\langle\hat{p}_{R}^{\dagger} \chi \mid \Psi\right\rangle & =\left\langle\chi \mid \hat{p}_{R} \Psi\right\rangle \\
& =\left\langle\hat{p}_{R} \chi \mid \Psi\right\rangle+i\left[\chi_{e}(0)^{*} \Psi_{o}(0)+\chi_{o}(0)^{*} \Psi_{e}(0)\right] .
\end{aligned}
$$

Next, we impose the boundary condition

$$
\Psi_{o}(0)=\lambda \Psi_{e}(0),
$$

which constrains the domain $D\left(\hat{p}_{R}\right)$. Inserting this relation in Eq. (31), the Hermiticity condition becomes

$$
\left[\chi_{e}(0)^{*} \lambda+\chi_{o}(0)^{*}\right] \Psi_{e}(0)=0 .
$$

Since $\Psi_{e}(0)$ can still take arbitrary values, one obtains

$$
\chi_{o}(0)=-\lambda^{*} \chi_{e}(0) \text {. }
$$

The operator $\hat{p}_{R}$ is self-adjoint if $D\left(\hat{p}_{R}^{\dagger}\right)=D\left(\hat{p}_{R}\right)$, which is true when $\lambda=-\lambda^{*}$, such that $\lambda \in i \mathbb{R}$. As a result, we obtain a 1-parameter family of self-adjoint extensions, characterized by the purely imaginary parameter $\lambda$. Since $\hat{p}_{R}$ is self-adjoint, it has a complete set of orthonormal eigenstates with corresponding real eigenvalues. The momentum eigenstates, which obey $\hat{p}_{R} \phi_{k}(x)=k \phi_{k}(x)$ with $k \in \mathbb{R}$, are given by

$$
\begin{aligned}
\phi_{k}(x) & =\frac{1}{\sqrt{2}}\left(\begin{array}{c}
\exp (i k x)+\sigma \exp (-i k x) \\
\exp (i k x)-\sigma \exp (-i k x)
\end{array}\right), \\
\sigma & =\frac{1-\lambda}{1+\lambda} \in U(1) .
\end{aligned}
$$


Like the momentum eigenstates $|k\rangle$ on the entire real axis, the states $\left|\phi_{k}\right\rangle$ on the half-line are orthonormalized to $\delta$ functions, i.e., $\left\langle\phi_{k^{\prime}} \mid \phi_{k}\right\rangle=2 \pi \delta\left(k-k^{\prime}\right)$.

\section{B. Embedding of $\hat{H}$ in $L^{2}\left(\mathbb{R}_{\geqslant 0}\right) \times \mathbb{C}^{2}$}

The search for a self-adjoint momentum operator that is strictly limited to the half-line has naturally put us into the Hilbert space $L^{2}\left(\mathbb{R}_{\geqslant 0}\right) \times \mathbb{C}^{2}$ of the doubly covered positive real axis. The double cover reflects the importance of even and odd lattice points in the underlying ultraviolet regularization at the level of the continuum description that emerges in the limit of vanishing lattice spacing. In order to apply the alternative concept for the momentum to the particle on the half-line, we must embed the original Hamiltonian $\hat{H}=-\frac{1}{2 m} \partial_{x}^{2}+V(x)$ with the self-adjoint extension parameter $\gamma$, which acts in $L^{2}\left(\mathbb{R}_{\geqslant 0}\right)$, into the doubled Hilbert space $L^{2}\left(\mathbb{R}_{\geqslant 0}\right) \times \mathbb{C}^{2}$. This is achieved by constructing the Hamiltonian

$$
\hat{H}(\mu)=\left(\begin{array}{cc}
-\frac{1}{2 m} \partial_{x}^{2}+V(x) & 0 \\
0 & -\frac{1}{2 m} \partial_{x}^{2}+V(x)
\end{array}\right)+\mu \hat{P}_{-} .
$$

Here $\hat{P}_{-}$projects on states $\Psi^{-}(x)$ with $\Psi_{o}^{-}(x)=-\Psi_{e}^{-}(x)$. In the underlying lattice theory, these states have energies at the lattice cutoff. In order to decouple them from the continuum theory, we take the limit $\mu \rightarrow \infty$. The complementary operator $\hat{P}_{+}$projects on the remaining states $\Psi^{+}(x)$ with $\Psi_{o}^{+}(x)=$ $\Psi_{e}^{+}(x)$, which have finite energy, i.e.,

$$
\begin{aligned}
\hat{P}_{ \pm} & =\frac{1}{2}\left(\begin{array}{cc}
1 & \pm 1 \\
\pm 1 & 1
\end{array}\right), \quad \hat{P}_{ \pm}^{2}=\hat{P}_{ \pm}, \quad \hat{P}_{+}+\hat{P}_{-}=\mathbb{1}, \\
\Psi(x) & =\Psi^{+}(x)+\Psi^{-}(x), \quad \Psi^{ \pm}(x)=\hat{P}_{ \pm} \Psi(x) .
\end{aligned}
$$

What is the most general boundary condition at $x=0$ for the extended Hamiltonian $\hat{H}(\mu)$ ? The linearity of quantum mechanics restricts us to writing

$$
\left(\begin{array}{c}
\Psi_{o}(0) \\
\partial_{x} \Psi_{o}(0)
\end{array}\right)=e^{i \eta}\left(\begin{array}{cc}
a & -b \\
-c & d
\end{array}\right)\left(\begin{array}{c}
\Psi_{e}(0) \\
\partial_{x} \Psi_{e}(0)
\end{array}\right) .
$$

Self-adjointness of $\hat{H}(\mu)$ again demands that the probability current density vanishes at the origin, $j(0)=0$. For 2-component wave functions the current density takes the form

$$
\begin{aligned}
j(x)= & \frac{1}{2 m i}\left[\Psi(x)^{*} \partial_{x} \Psi(x)-\partial_{x} \Psi(x)^{*} \Psi(x)\right] \\
= & \frac{1}{2 m i}\left[\Psi_{e}(x)^{*} \partial_{x} \Psi_{e}(x)-\partial_{x} \Psi_{e}(x)^{*} \Psi_{e}(x)\right. \\
& \left.+\Psi_{o}(x)^{*} \partial_{x} \Psi_{o}(x)-\partial_{x} \Psi_{o}(x)^{*} \Psi_{o}(x)\right] .
\end{aligned}
$$

Using $j(0)=0$, it is straightforward to derive the conditions $a, b, c, d \in \mathbb{R}$ and $a d-b c=-1$. Together with $\eta$, these are four independent parameters, which define a family of selfadjoint extensions. In order to correctly embed the original Hamiltonian $\hat{H}$, the boundary condition must support the finite-energy states with $\Psi_{o}^{+}(x)=\Psi_{e}^{+}(x)$, which requires

$$
e^{i \eta}=1, \quad a=1, \quad b=0, \quad d=-1 .
$$

Using these specific parameters, Eq. (38) reduces to

$$
-\frac{c}{2} \Psi^{+}(0)-\partial_{x} \Psi^{+}(0)=0, \quad \Psi^{-}(0)=0 .
$$

We now identify $\Psi^{+}(x)$ with the wave functions in the original Hilbert space $L^{2}\left(\mathbb{R}_{\geqslant 0}\right)$. Putting $\gamma=-c / 2$, Eq. (41) reduces to the Robin boundary condition of Eq. (3), while the wave functions $\Psi^{-}(x)$ obey Dirichlet boundary conditions. By construction, in the limit $\mu \rightarrow \infty$ the Hamiltonian $\hat{H}(\mu)$ has the same finite-energy spectrum as the original Hamiltonian $\hat{H}$. The corresponding eigenstates of $\hat{H}(\mu)$ are just identical copies of the original eigenstates of $\hat{H}$ (renormalized by $\frac{1}{\sqrt{2}}$ ) in the upper and lower component of the 2-component wave function.

\section{Momentum measurements on $\mathbb{R}_{\geqslant 0}$}

We are now ready to apply the alternative concept of momentum to the particle on the half-line. An original wave function $\psi(x) \in L^{2}\left(\mathbb{R}_{\geqslant 0}\right)$ is trivially embedded in the doubled Hilbert space $L^{2}\left(\mathbb{R}_{\geqslant 0}\right) \times \mathbb{C}^{2}$ as

$$
\Psi^{+}(x)=\left(\begin{array}{l}
\Psi_{e}^{+}(x) \\
\Psi_{o}^{+}(x)
\end{array}\right)=\frac{1}{\sqrt{2}}\left(\begin{array}{l}
\psi(x) \\
\psi(x)
\end{array}\right) .
$$

The probability to measure a momentum value $k$ is determined by the amplitude

$$
\begin{aligned}
\left\langle\phi_{k} \mid \Psi^{+}\right\rangle & =\frac{1}{2} \int_{0}^{\infty} d x\left(\begin{array}{l}
\exp (i k x)+\sigma \exp (-i k x) \\
\exp (i k x)-\sigma \exp (-i k x)
\end{array}\right)^{\dagger}\left(\begin{array}{l}
\psi(x) \\
\psi(x)
\end{array}\right) \\
& =\int_{-\infty}^{\infty} d x \exp (-i k x) \psi(x) \theta(x)=\langle k \mid \psi\rangle
\end{aligned}
$$

Remarkably, $\langle k \mid \psi\rangle$ is just the amplitude that determines the probability to obtain the value $k$ in a measurement of the standard momentum operator $\hat{p}=-i \partial_{x}$ that is self-adjoint only over the entire real axis. Here, using the step function $\theta(x)$, the wave function $\psi(x)$ has been trivially extended to the negative real axis. As a result, both the standard and the alternative concept of momentum yield the same probability distributions for the measurement results. However, the two concepts project onto different states after the measurements. In particular, while a standard momentum measurement puts the particle also onto the negative real axis, the alternative concept is strictly limited to the half-line; i.e., also after the measurement the particle remains on the positive real axis. Still, also with the alternative concept a momentum measurement transfers an infinite amount of energy to the particle. This is because the momentum eigenstate $\phi_{k}(x)$, onto which a measurement that results in the value $k$ projects, not only has the finite-energy component $\phi_{k}^{+}(x)=\frac{1}{\sqrt{2}} \exp (i k x)$ but also the component $\phi_{k}^{-}(x)=\frac{1}{\sqrt{2}} \sigma \exp (-i k x)$, whose energy diverges in the limit $\mu \rightarrow \infty$. Interestingly, the purely imaginary selfadjoint extension parameter $\lambda$, which determines $\sigma=(1-$ $\lambda) /(1+\lambda) \in U(1)$, does not affect the probability to obtain a certain momentum measurement result.

As we will see later, the standard and the alternative concept for the momentum no longer result in the same measurement results when one considers a particle that is confined to an interval [9]. In that case, a standard momentum measurement catapults the particle outside of the interval and results in a continuous momentum value. The alternative concept, on the other hand, yields discrete momentum values and leaves the particle inside the interval after a measurement. 


\section{Canonical quantization on the half-line $\mathbb{R}_{\geqslant 0}$}

As we have seen in Eq. (10), in canonical quantization the unitary operator $U_{a}=\exp (i \hat{p} a)$ with $\hat{p}=-i \partial_{x}$ performs a coordinate shift, $U_{a} \Psi(x)=\Psi(x+a)$, while the operator $\widetilde{U}_{q}=$ $\exp (i q \hat{x})$ with $\hat{x}=i \partial_{p}$ performs a momentum shift, $\widetilde{U}_{q} \widetilde{\Psi}(p)=$ $\widetilde{\Psi}(p-q)$ [cf. Eq. (12)]. What is the unitary operator $\widetilde{V}_{q}$ that shifts the eigenstates $\phi_{k}(x)$ of the self-adjoint momentum operator on the half-line, $\hat{p}_{R}=-i \sigma_{1} \partial_{x}$, to $\phi_{k+q}(x)$ ? We construct

$$
\begin{aligned}
\phi_{k+q}(x)= & \frac{1}{\sqrt{2}}\left(\begin{array}{c}
\exp [i(k+q) x]+\sigma \exp [-i(k+q) x] \\
\exp [i(k+q) x]-\sigma \exp [-i(k+q) x]
\end{array}\right) \\
= & \left(\begin{array}{cc}
\cos (q x) & i \sin (q x) \\
i \sin (q x) & \cos (q x)
\end{array}\right) \\
& \times \frac{1}{\sqrt{2}}\left(\begin{array}{cc}
\exp (i k x)+\sigma \exp (-i k x) \\
\exp (i k x)-\sigma \exp (-i k x)
\end{array}\right) \\
= & \widetilde{V}_{q} \phi_{k}(x) \Rightarrow \\
\widetilde{V}_{q}= & \left(\begin{array}{ll}
\cos (q x) & i \sin (q x) \\
i \sin (q x) & \cos (q x)
\end{array}\right)=\exp \left(i q \hat{x}_{R}\right), \hat{x}_{R}=\sigma_{1} x .
\end{aligned}
$$

Not surprisingly, the conjugate coordinate to the momentum $\hat{p}_{R}=-i \sigma_{1} \partial_{x}$ is $\hat{x}_{R}=\sigma_{1} x$. Indeed, the two operators obey the canonical commutation relation $\left[\hat{x}_{R}, \hat{p}_{R}\right]=i$. According to the Stone-von Neumann theorem [43-46], this implies that $\hat{x}_{R}$ and $\hat{p}_{R}$ are unitarily equivalent to the standard coordinate $\hat{x}$ and momentum $\hat{p}$. While this is true mathematically, if not properly interpreted, it might be physically misleading. In particular, while the eigenstates of $\hat{x}$ describe positions on the entire real axis, the eigenstates of $\hat{x}_{R}$ describe a double cover of the half-line. Hence, the situations are, in fact, physically distinct, but, as we will see explicitly below, indeed mathematically related by a unitary transformation. This may still seem surprising, since $\hat{p}_{R}$ possesses the self-adjoint extension parameter $\lambda \in i \mathbb{R}$ which characterizes the domain $D\left(\hat{p}_{R}\right)$, while the standard momentum operator does not. However, two operators $\hat{p}_{R}$, which are endowed with different self-adjoint extension parameters $\lambda$ and $\lambda^{\prime}$, are indeed related by a unitary transformation

$$
\begin{aligned}
& W=\exp \left(i \omega \sigma_{1}\right)=\left(\begin{array}{cc}
\cos \omega & i \sin \omega \\
i \sin \omega & \cos \omega
\end{array}\right), \\
& W\left(-i \sigma_{1} \partial_{x}\right) W^{\dagger}=-i \sigma_{1} \partial_{x},
\end{aligned}
$$

which leaves the differential expression for $\hat{p}_{R}$ invariant. The boundary condition $\Psi_{o}(0)=\lambda \Psi_{e}(0)$ then turns into

$$
\begin{aligned}
\left(\begin{array}{l}
\Psi_{e}^{\prime}(0) \\
\Psi_{o}^{\prime}(0)
\end{array}\right) & =W\left(\begin{array}{l}
\Psi_{e}(0) \\
\Psi_{o}(0)
\end{array}\right)=\left(\begin{array}{c}
\cos \omega \Psi_{e}(0)+i \sin \omega \Psi_{o}(0) \\
i \sin \omega \Psi_{e}(0)+\cos \omega \Psi_{o}(0)
\end{array}\right) \\
& =\left(\begin{array}{c}
(\cos \omega+\lambda i \sin \omega) \Psi_{e}(0) \\
(i \sin \omega+\lambda \cos \omega) \Psi_{e}(0)
\end{array}\right) \Rightarrow \\
\Psi_{o}^{\prime}(0) & =\frac{i \sin \omega+\lambda \cos \omega}{\cos \omega+\lambda i \sin \omega} \Psi_{e}^{\prime}(0)=\lambda^{\prime} \Psi_{e}^{\prime}(0)
\end{aligned}
$$

As a result, two different operators $\hat{p}_{R}$, which are associated with the self-adjoint extension parameters $\lambda$ and $\lambda^{\prime}$, are related by the unitary transformation $W$ with

$$
\begin{aligned}
\tan \omega & =i \frac{\lambda-\lambda^{\prime}}{1-\lambda \lambda^{\prime}}, \\
\sigma^{\prime} & =\frac{1-\lambda^{\prime}}{1+\lambda^{\prime}}=\exp (-2 i \omega) \frac{1-\lambda}{1+\lambda}=\exp (-2 i \omega) \sigma .
\end{aligned}
$$

Let us again denote the eigenstates of $\hat{x}$ as $|x\rangle$, such that $\hat{x}|x\rangle=x|x\rangle$. Here we limit ourselves to the positive real axis with $x>0$. The eigenstates of $\hat{x}_{R}=\sigma_{1} x$ are then given by

$$
\begin{aligned}
& \hat{x}_{R}|x,+\rangle=x|x,+\rangle,|x,+\rangle=\frac{1}{\sqrt{2}}\left(\begin{array}{c}
|x\rangle \\
|x\rangle
\end{array}\right), \\
& \hat{x}_{R}|x,-\rangle=-x|x,-\rangle,|x,-\rangle=\frac{1}{\sqrt{2}}\left(\begin{array}{c}
|x\rangle \\
-|x\rangle
\end{array}\right) .
\end{aligned}
$$

The eigenstates $|x,+\rangle$ have a positive eigenvalue $x>0$ and belong to the finite-energy sector, i.e., $\hat{P}_{+}|x,+\rangle=|x,+\rangle$, while the eigenstates $|x,-\rangle$ have a negative eigenvalue $-x$ and obey $\hat{P}_{-}|x,-\rangle=|x,-\rangle, \hat{P}_{+}|x,-\rangle=0$.

How does the operator $V_{a}=\exp \left(i \hat{p}_{R} a\right)$ act on the eigenstates $|x, \pm\rangle$ ? We obtain

$$
\begin{aligned}
V_{a}|x, \pm\rangle & =\frac{1}{2 \pi} \int_{-\infty}^{\infty} d k \exp \left(i \hat{p}_{R} a\right)\left|\phi_{k}\right\rangle\left\langle\phi_{k} \mid x, \pm\right\rangle \\
& =\frac{1}{2 \pi} \int_{-\infty}^{\infty} d k \exp (i k a)\left|\phi_{k}\right\rangle\left\langle\phi_{k} \mid x, \pm\right\rangle, \\
\left\langle x,+\mid \phi_{k}\right\rangle & =\frac{1}{2}(1,1)\left(\begin{array}{l}
\exp (i k x)+\sigma \exp (-i k x) \\
\exp (i k x)-\sigma \exp (-i k x)
\end{array}\right) \\
& =\exp (i k x), \\
\left\langle x,-\mid \phi_{k}\right\rangle & =\frac{1}{2}(1,-1)\left(\begin{array}{c}
\exp (i k x)+\sigma \exp (-i k x) \\
\exp (i k x)-\sigma \exp (-i k x)
\end{array}\right) \\
& =\sigma \exp (-i k x),
\end{aligned}
$$

which implies

$$
\begin{aligned}
& V_{a}|x,+\rangle=|x-a,+\rangle, \text { for } 0 \leqslant \mathrm{x}-\mathrm{a}, \\
& V_{a}|x,+\rangle=\sigma|a-x,-\rangle, \text { for } \mathrm{x}-\mathrm{a}<0, \\
& V_{a}|x,-\rangle=|x+a,-\rangle, \text { for } 0 \leqslant \mathrm{x}+\mathrm{a}, \\
& V_{a}|x,-\rangle=\sigma^{*}|-x-a,+\rangle, \text { for } \mathrm{x}+\mathrm{a}<0 .
\end{aligned}
$$

Writing $\hat{x}_{R}\left|x_{R}\right\rangle=x_{R}\left|x_{R}\right\rangle, x_{R} \in \mathbb{R}$, we identify $\left|x_{R}\right\rangle=\left|x_{R},+\right\rangle$ for $x_{R}>0$, and $\left|x_{R}\right\rangle=\left|-x_{R},-\right\rangle$ for $x_{R}<0$. Equation (50) then implies that, up to a phase $\sigma$ or $\sigma^{*}, V_{a}$ shifts the eigenstates of $\hat{x}_{R}$ from $\left|x_{R}\right\rangle$ to $\left|x_{R}-a\right\rangle$. Independently of $\sigma$, as a consequence of the Heisenberg algebra $\left[\hat{x}_{R}, \hat{p}_{R}\right]=i$, one obtains the Weyl group relation

$$
V_{a} \widetilde{V}_{q}=\exp (\text { iqa }) \widetilde{V}_{q} V_{a}
$$

The action of the operators $V_{a}$ and $\widetilde{V}_{q}$ is illustrated in Fig. 2. The operator $V_{a}=\exp \left(i \hat{p}_{R} a\right)$ transports states $|x,+\rangle$ in the finite-energy sector to the left, and states $|x,-\rangle$, whose energy is ultraviolet-sensitive, to the right. When a state $|x,+\rangle$ is transported beyond the origin (at $x=0$ ), it turns into the state $|a-x,-\rangle$.

For mathematical purposes, the Weyl groups are more convenient than the corresponding Heisenberg algebras because unitary operators are bounded while the corresponding self-adjoint operators are not. Based upon the Weyl groups 


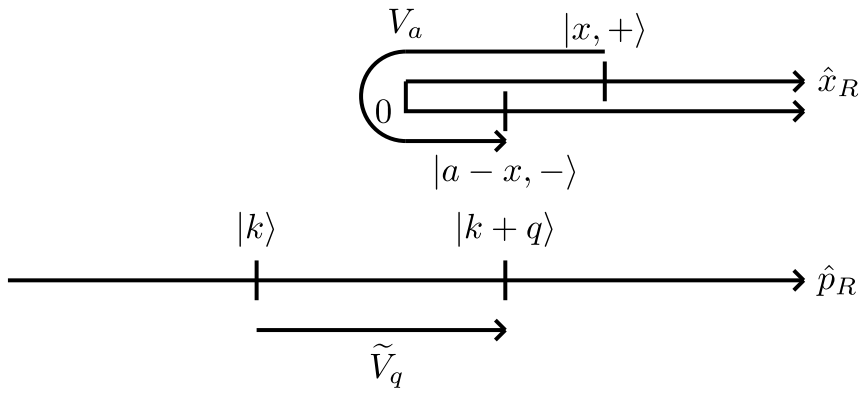

FIG. 2. Action of the translation operators $V_{a}=\exp \left(i \hat{p}_{R} a\right)$ and $\widetilde{V}_{q}=\exp \left(i q \hat{x}_{R}\right)$ on the position and momentum eigenstates for the half-line $\mathbb{R}_{\geqslant 0}$.

of Eq. (15) and Eq. (51), the Stone-von Neumann theorem guarantees the unitary equivalence of $\hat{x}_{R}$ and $\hat{p}_{R}$ with $\hat{x}$ and $\hat{p}$. The corresponding unitary transformation $U$ is given by

$$
\begin{aligned}
& U V_{a} U^{\dagger}=U_{a}, \quad U \widetilde{V}_{q} U^{\dagger}=\widetilde{U}_{q}, \\
& U \hat{x}_{R} U^{\dagger}=\hat{x}, \quad U|x,+\rangle=|x\rangle, \quad U|x,-\rangle=\sigma^{*}|-x\rangle, \\
& U \hat{p}_{R} U^{\dagger}=\hat{p}, \quad U\left|\phi_{k}\right\rangle=|k\rangle .
\end{aligned}
$$

We would like to stress again that this mathematical unitary transformation relates two physically very different situations. In particular, while the states $|x,+\rangle$ (with $x>0$ ) in the finiteenergy sector are mapped to position eigenstates $|x\rangle$ on the positive real axis, the states $|x,-\rangle$ (again with $x>0$ ), which are mapped to the states $|-x\rangle$ on the negative real axis, actually reside on the positive half-line, but have ultravioletsensitive energies and are removed from the spectrum of the Hamiltonian $\hat{H}(\mu)$ in the limit $\mu \rightarrow \infty$.

\section{E. The classical limit}

Until now we have used the term quantization-be it canonical or affine-without even starting from a classical theory. Since quantum physics is more fundamental than classical physics, this way to proceed is not unreasonable. Until now, we have shown that our alternative concept gives rise to a self-adjoint momentum operator $\hat{p}_{R}=-i \sigma_{1} \partial_{x}$ that obeys the canonical commutation relation, $\left[\hat{x}_{R}, \hat{p}_{R}\right]=i$, with the corresponding coordinate $\hat{x}_{R}=\sigma_{1} x$. We now consider the classical limit in order to demonstrate that the alternative concept still makes sense when commutators are replaced by Poisson brackets.

Let us perform a unitary transformation $D$ that diagonalizes $\hat{x}_{R}, \hat{p}_{R}$, and $\hat{H}(\mu)$ such that

$$
\begin{aligned}
D \sigma_{1} D^{\dagger} & =\sigma_{3}, \quad D=\frac{1}{\sqrt{2}}\left(\begin{array}{cc}
1 & 1 \\
1 & -1
\end{array}\right), \\
\hat{x}_{R}^{\prime} & =D \hat{x}_{R} D^{\dagger}=x \sigma_{3}=\left(\begin{array}{cc}
x & 0 \\
0 & -x
\end{array}\right), \quad x \in \mathbb{R}_{\geqslant 0}, \\
\hat{p}_{R}^{\prime} & =D \hat{p}_{R} D^{\dagger}=-i \sigma_{3} \partial_{x}=-i\left(\begin{array}{cc}
\partial_{x} & 0 \\
0 & -\partial_{x}
\end{array}\right), \\
\hat{H}(\mu)^{\prime} & =D \hat{H}(\mu) D^{\dagger} \\
& =\left(\begin{array}{cc}
-\frac{1}{2 m} \partial_{x}^{2}+V(x) & -\frac{1}{2 m} \partial_{x}^{2}+V(x)+\mu
\end{array}\right) .
\end{aligned}
$$

The positive eigenvalues of $\hat{x}_{R}$ correspond to physical points $x \in \mathbb{R}_{\geqslant 0}$ on the half-line on which finite-energy states are located. The negative eigenvalues $-x$ of $\hat{x}_{R}$, on the other hand, are not associated with the negative real axis, but host states with energies at the ultraviolet cutoff scale, which are removed in the limit $\mu \rightarrow \infty$.

A similar mathematical description arises when one applies the standard concept of momentum. In that case, one regularizes the problem by assigning a potential $V(x<0)=V_{0}$ to points on the negative real axis, and ultimately taking the limit $V_{0} \rightarrow \infty$. At a formal level $V_{0}$ then corresponds to $\mu$, but the physical interpretation is very different. In fact, when one applies the alternative concept for the momentum, the particle is strictly limited to the positive real axis, even after a momentum measurement.

In any case, one can now replace the commutation relation $\left[\hat{x}_{R}^{\prime}, \hat{p}_{R}^{\prime}\right]=i$ by the classical Poisson bracket relation $\{x, p\}=$ 1 and replace the Hamiltonian $\hat{H}(\mu)^{\prime}$ in the limit $\mu \rightarrow \infty$ by the classical Hamilton function $\mathcal{H}(x, p)=\frac{p^{2}}{2 m}+V(x)$. The hard wall at $x=0$ arises as a consequence of $\mu \rightarrow \infty$, thus leading to the standard motion of a classical particle that gets reflected at the origin. The self-adjoint extension parameters $\lambda$ and $\gamma$ leave no trace in the classical limit. In particular, unlike the quantum particle, the classical particle cannot be bound to the reflecting wall at $x=0$.

\section{F. Subtleties with commutators and operator domains}

Canonical quantization (as defined in the introduction) usually starts from a classical theory with the Poisson bracket relation $\{x, p\}=1$, which is then promoted to the commutation relation $[\hat{x}, \hat{p}]=i$ between self-adjoint operators $\hat{x}$ and $\hat{p}$. Until now, it was assumed that on the half-line canonical quantization fails, simply because in this case no self-adjoint momentum operator exists for $L^{2}\left(\mathbb{R}_{\geqslant 0}\right)$. As we have seen, a satisfactory self-adjoint momentum operator $\hat{p}_{R}=-i \sigma_{1} \partial_{x}$ as well as its canonically conjugate coordinate $\hat{x}_{R}=\sigma_{1} x$ (with $x \geqslant 0)$ indeed exist in the doubled Hilbert space $L^{2}\left(\mathbb{R}_{\geqslant 0}\right) \times$ $\mathbb{C}^{2}$. The operator $\hat{p}_{R}$ is endowed with the self-adjoint extension parameter $\lambda$, and is thus not uniquely defined. However, as we have seen in Eq. (46), in accordance with the Stone-von Neumann theorem [43-46], two operators $\hat{p}_{R}$, which are associated with two different self-adjoint extension parameters $\lambda$ and $\lambda^{\prime}$, are, in fact, unitarily equivalent.

Following the canonical quantization procedure, one replaces the classical Hamilton function $\mathcal{H}(x, p)=\frac{p^{2}}{2 m}+V(x)$ with a Hamilton operator $\hat{H}$ by replacing $x$ with $\hat{x}$ and $p$ with $\hat{p}$. When one replaces the classical kinetic energy $\mathcal{T}=\frac{p^{2}}{2 m}$ with the operator

$$
\hat{T}=\frac{\hat{p}_{R}^{2}}{2 m}=\frac{1}{2 m}\left(-i \sigma_{1} \partial_{x}\right)^{2}=-\frac{1}{2 m} \partial_{x}^{2} \mathbb{1},
$$

at least at the formal level of differential expressions one obtains the correct kinetic energy operator that enters the Hamiltonian $\hat{H}(\mu)$ of Eq. (36).

At a superficial level one might conclude that $\hat{T}$ and $\hat{p}_{R}$ commute. However, $\hat{T}$ is self-adjoint in $L^{2}\left(\mathbb{R}_{\geqslant 0}\right) \times \mathbb{C}^{2}$ only if it is endowed with its own self-adjoint extension parameters which characterize the domain $D(\hat{T})$. The most general 
self-adjoint extension of $\hat{T}$ is characterized by Eq. (38), which contains four independent parameters: $\eta, a, b, c, d \in \mathbb{R}$ subject to the constraint $a d-b c=-1$. However, for the reasons explained above, here we are only interested in the special case $\eta=0, a=1, b=0, c=-2 \gamma, d=-1$. For general finite $\gamma<\infty$, one then has $\Psi_{e}(0)=\Psi_{o}(0)$, which is inconsistent with $\Psi_{o}(0)=\lambda \Psi_{e}(0)$, because $\lambda \in i \mathbb{R}$ is purely imaginary. As a result, the domains of the momentum operator and the kinetic energy operator are not the same, $D\left(\hat{p}_{R}\right) \neq$ $D(\hat{T})$. Only when we choose Dirichlet boundary conditions (which correspond to $\gamma \rightarrow \infty$ ), the domain of $\hat{T}$ is characterized by $\Psi_{e}(0)=\Psi_{o}(0)=0$, which is automatically consistent with $\Psi_{o}(0)=\lambda \Psi_{e}(0)$. Hence, in this special case, $D(\hat{T}) \subset$ $D\left(\hat{p}_{R}\right)$. This implies that an application of $\hat{p}_{R}$ on a wave function $\hat{T} \Psi(x) \in D(\hat{T})$ is still possible and $\hat{p}_{R} \hat{T} \Psi(x) \in D\left(\hat{p}_{R}\right)$. On the other hand, an application of $\hat{T}$ on $\hat{p}_{R} \Psi(x) \in D\left(\hat{p}_{R}\right)$ is possible only if $\hat{p}_{R} \Psi(x) \in D(\hat{T})$. Hence, the commutator $\hat{p}_{R} \hat{T}-\hat{T} \hat{p}_{R}$ cannot act on all wave functions in $D\left(\hat{p}_{R}\right)$ or $D(\hat{T})$, and hence has only a limited formal meaning. As a result, although the operators $\hat{p}_{R}$ and $\hat{T}$ seem to commute at the superficial level of differential expressions, they do not have common eigenfunctions.

Let us return to the operators $\hat{x}$ and $\hat{d}=-i\left(\frac{1}{2}+x \partial_{x}\right)$ that we encountered in affine quantization and consider them together with the operators $\hat{x}_{R}=\sigma_{1} x$ and $\hat{p}_{R}=-i \sigma_{1} \partial_{x}$ that we used in canonical quantization. Among those, only the domain $D\left(\hat{p}_{R}\right)$ is further restricted by the condition $\Psi_{o}(0)=\lambda \Psi_{e}(0)$ with $\lambda \in i \mathbb{R}$. First of all,

$$
\hat{x}_{R} \Psi(x)=\left(\begin{array}{ll}
0 & x \\
x & 0
\end{array}\right)\left(\begin{array}{l}
\Psi_{e}(x) \\
\Psi_{o}(x)
\end{array}\right)=\left(\begin{array}{l}
x \Psi_{o}(x) \\
x \Psi_{e}(x)
\end{array}\right),
$$

which does not lead out of $D\left(\hat{p}_{R}\right)$, because $x \Psi_{e}(x)=$ $\lambda x \Psi_{o}(x)=0$ at $x=0$. Trivially embedding $\hat{x}$ into the doubled Hilbert space, we also obtain

$$
\left(\begin{array}{ll}
\hat{x} & 0 \\
0 & \hat{x}
\end{array}\right)\left(\begin{array}{l}
\Psi_{e}(x) \\
\Psi_{o}(x)
\end{array}\right)=\left(\begin{array}{l}
x \Psi_{e}(x) \\
x \Psi_{o}(x)
\end{array}\right)
$$

which does not lead out of $D\left(\hat{p}_{R}\right)$ either. Finally, by also embedding $\hat{d}$ into the doubled Hilbert space, one obtains

$$
\left(\begin{array}{ll}
\hat{d} & 0 \\
0 & \hat{d}
\end{array}\right)\left(\begin{array}{l}
\Psi_{e}(x) \\
\Psi_{o}(x)
\end{array}\right)=\left(\begin{array}{l}
-i\left(\frac{1}{2}+x \partial_{x}\right) \Psi_{e}(x) \\
-i\left(\frac{1}{2}+x \partial_{x}\right) \Psi_{o}(x)
\end{array}\right) .
$$

Putting $x=0$, we then find

$$
\begin{aligned}
-i\left(\frac{1}{2}+x \partial_{x}\right) \Psi_{o}(0) & =-\frac{i}{2} \Psi_{o}(0)=-\frac{i}{2} \lambda \Psi_{e}(0) \\
& =-\lambda i\left(\frac{1}{2}+x \partial_{x}\right) \Psi_{e}(0) .
\end{aligned}
$$

We thus conclude that $\hat{d}$ does not lead out of $D\left(\hat{p}_{R}\right)$ either. Since $\hat{x}, \hat{x}_{R}$, and $\hat{d}$ are not subject to further domain restrictions of their own, limiting ourselves to wave functions $\Psi(x) \in$ $D\left(\hat{p}_{R}\right)$, the following commutators are indeed not just formal expressions, but mathematically and physically meaningful,

$$
\begin{aligned}
& {\left[\hat{x}, \hat{x}_{R}\right]=0, \quad[\hat{x}, \hat{d}]=i \hat{x}, \quad\left[\hat{x}, \hat{p}_{R}\right]=i \sigma_{1},} \\
& {\left[\hat{x}_{R}, \hat{d}\right]=\left[\sigma_{1} x,-i x \partial_{x}\right]=i \sigma_{1} x=i \hat{x}_{R}, \quad\left[\hat{x}_{R}, \hat{p}_{R}\right]=i,} \\
& {\left[\hat{p}_{R}, \hat{d}\right]=\left[-i \sigma_{1} \partial_{x},-i x \partial_{x}\right]=-\sigma_{1} \partial_{x}=-i \hat{p}_{R} .}
\end{aligned}
$$

This implies that affine and canonical quantization are fully consistent with each other and can be implemented simultaneously.

\section{G. Summary of the canonical quantization procedure}

To summarize, the resulting canonical quantization procedure consists of the following steps:

(i) Select a classical system characterized by coordinates $x$ and canonically conjugate momenta $p$ associated with a classical Hamilton function $\mathcal{H}(x, p)$ as well as with some observables $\mathcal{A}(x, p)$.

(ii) Identify an appropriate Hilbert space in which to realize the time evolution and the measurements for a corresponding quantum system.

(iii) Replace classical Poisson bracket relations by formal commutation relations.

(iv) Realize the commutation relations by differential expressions.

(v) Extend the differential expressions to self-adjoint operators.

(vi) Make a specific choice of self-adjoint extension parameters and thus of the corresponding operator domains.

(vii) Return to the formal commutation relations, investigate whether they are affected by domain incompatibilities, and take this into account properly.

(viii) If the canonical commutation relation between position and momentum itself is compromised by domain incompatibilities, try to replace the position or the momentum operator by more appropriate operators that are unaffected by such subtleties.

As we have seen, the identification of an appropriate Hilbert space may be nontrivial and may depend on the set of observables to be measured. In particular, for the particle on the half-line the time evolution driven by the Hamiltonian $\hat{H}$ can be realized in the Hilbert space $L^{2}\left(\mathbb{R}_{\geqslant 0}\right)$. However, in order to consider momentum measurements, the Hilbert space must be doubled to $L^{2}\left(\mathbb{R}_{\geqslant 0}\right) \times \mathbb{C}^{2}$ and the Hamiltonian must be extended accordingly to $\hat{H}(\mu)$. In the absence of subtle Hilbert space or domain incompatibility issues, all relevant commutators correctly reflect the relations between the various operators, and the procedure outlined above simplifies considerably. When such subtleties do arise, on the other hand, naively applying the commutation relations that result from classical Poisson brackets may lead to wrong results. This would happen, for example, if one would not realize that the self-adjoint operators $\hat{p}_{R}$ and $\hat{T}$ do not commute, although the corresponding differential expressions seem to suggest this. In such a case, it is necessary to properly address the subtleties, which is possible using the well-developed mathematical theory that was initiated by von Neumann [27].

The remaining steps for addressing the quantum dynamics are the usual ones, but they are strongly affected by the selected operator domains:

(i) Employ the Hamiltonian to determine the wave function that solves the Schrödinger equation.

(ii) Solve the eigenvalue problem for the operators that describe observables to be measured.

(iii) Project the wave function on the eigenfunction of an observable in order to predict the probability to measure the corresponding eigenvalue. 
It should be stressed that there is absolutely nothing wrong with affine quantization applied to the half-line. In view of our construction, it is just no longer the only quantization procedure that is available in this case.

\section{H. Is canonical quantization a fine quantization for the half-line?}

Is there anything wrong with the above canonical quantization procedure applied to the half-line? We will now argue that this admittedly somewhat subtle procedure is completely fine, and, in fact, very natural in a continuous space with sharp boundaries. First of all, in order to motivate the construction of $\hat{p}_{R}$ in the first place, in [9] we have introduced an ultraviolet regularization by replacing the continuum with a spatial lattice with $N$ points. The resulting Hilbert space is then $N$ dimensional. In a finite-dimensional Hilbert space, there is no difference between Hermiticity and self-adjointness, and one need not worry about the domains of operators. The lattice variant of the momentum operator $\hat{p}_{R}$ results from a symmetrized forward-backward lattice derivative that extends over two lattice spacings. The lattice variant of the kinetic energy operator $\hat{T}$ is described by a standard finite-difference lattice version of a second derivative. The low-energy physics of the lattice system does not require an extension of the Hilbert space and does not depend on any operator domain issues. If we interpret the lattice spacing as mimicking a shortest relevant physical length scale, such as a crystal lattice spacing in a quantum dot, or the Planck length in an interval of extra-dimensional space, every Hermitian operator would automatically be self-adjoint and could act in the entire Hilbert space. In particular, the mathematical subtleties related to operator domains would have no effect on the physics.

However, also from a physics point of view, it is desirable to derive a low-energy effective description of the underlying lattice physics, by taking the continuum limit of vanishing lattice spacing. This would have been straightforward if we would care only about the spectrum of the Hamiltonian. In fact, one could then stay within the Hilbert space $L^{2}\left(\mathbb{R}_{\geqslant 0}\right)$. Since we also care about the momentum operator, the situation is more subtle. In particular, the crucial concept of even and odd lattice points naturally leads to the doubled Hilbert space $L^{2}\left(\mathbb{R}_{\geqslant 0}\right) \times \mathbb{C}^{2}$. As we have seen, the embedding of the original Hamiltonian $\hat{H}$ into this framework leads to the Hamiltonian $\hat{H}(\mu)$ with the specific self-adjoint extension parameters of Eq. (40). The limit $\mu \rightarrow \infty$ removes those states from the finite-energy spectrum whose lattice variants have energies at the cutoff scale. This inevitably leads to the different operator domains, $D\left(\hat{p}_{R}\right) \neq D(\hat{H}(\mu))$.

While the resulting canonical quantization procedure may seem unnecessarily complicated, it is the price a physicist has to pay for using a low-energy continuum description in an infinite-dimensional Hilbert space. This description is, in fact, mathematically quite elegant, but admittedly not completely straightforward. A straightforward alternative description could stay on the lattice without taking the continuum limit. However, such a formulation is not very transparent, it leads to more complicated variants of the Schrödinger equation, and it does not correspond to the standard quantum mechanical continuum description. We suggest that it is worth familiarizing oneself with the differences between Hermiticity and self-adjointness, and thus with the issues related to operator domains, even if this may require an expansion of the sometimes prevailing practices in quantum mechanics.

\section{CANONICAL QUANTIZATION IN AN INTERVAL AND ON A CIRCLE}

In this section, we apply the alternative concept for the momentum to an interval, which allows us to extend canonical quantization to that case as well. We then relate the results to the well-understood situation on a circle.

\section{A. Canonical quantization in the interval $[0, L]$}

In [9] we have introduced the alternative concept for the momentum operator in an interval $\left[-\frac{L}{2}, \frac{L}{2}\right]$. In that work we have only briefly touched upon the corresponding algebra that relates the finite-volume momentum to its corresponding conjugate coordinates. Here we consider this problem in more detail. We shift the interval to $[0, L]$ in order to be able to recover the half-line in the limit $L \rightarrow \infty$.

In an interval, the domain $D\left(\hat{p}_{R}\right)$ of the finite-volume momentum operator $\hat{p}_{R}$ is characterized by two self-adjoint extension parameters, $\lambda$ and $\lambda_{L}$, associated with the two ends of the interval. In the interval $[0, L]$ the boundary conditions are

$$
\Psi_{o}(0)=\lambda \Psi_{e}(0), \quad \Psi_{o}(L)=\lambda_{L} \Psi_{e}(L), \quad \lambda, \lambda_{L} \in i \mathbb{R}_{\geqslant 0} .
$$

The corresponding momentum eigenfunctions $\phi_{k}(x)$ are still given by Eq. (35). However, in contrast to the half-line, in the interval the momentum $k$ is quantized and obeys the condition

$$
\exp (2 i k L)=\frac{(1-\lambda)\left(1+\lambda_{L}\right)}{(1+\lambda)\left(1-\lambda_{L}\right)}=\sigma \sigma_{L}^{*}=\exp (i \theta) .
$$

For $\lambda=\lambda_{L}$ this leads to $k=\frac{\pi n}{L}, n \in \mathbb{Z}$, while in general

$$
k=\frac{\pi}{L}\left(n+\frac{\theta}{2 \pi}\right), n \in \mathbb{Z} .
$$

Adjacent momentum eigenvalues are hence separated by $\frac{\pi}{L}$. The self-adjoint extension parameter $\theta$ is invariant against the unitary transformation $W$ of Eq. (45) because

$$
\begin{aligned}
\exp \left(i \theta^{\prime}\right) & =\sigma^{\prime}{\sigma_{L}^{\prime *}}^{*}=\exp (-2 i \omega) \sigma \exp (2 i \omega) \sigma_{L}^{*} \\
& =\sigma \sigma_{L}{ }^{*}=\exp (i \theta) .
\end{aligned}
$$

Here we have used Eqs. (61) and (47).

What is the unitary operator that translates the momentum eigenfunctions by $\frac{\pi}{L}$ ? Using Eq. (44), which is still applicable in the interval, we obtain

$$
\begin{aligned}
& \phi_{k+\pi / L}(x)=\widetilde{V}_{\pi / L} \phi_{k}(x), \\
& \widetilde{V}_{\pi / L}=\exp \left(i \frac{\pi}{L} \hat{x}_{R}\right)=\left(\begin{array}{cc}
\cos \frac{\pi x}{L} & i \sin \frac{\pi x}{L} \\
i \sin \frac{\pi x}{L} & \cos \frac{\pi x}{L}
\end{array}\right) .
\end{aligned}
$$

The fact that $\widetilde{V}_{\pi / L}$ acts as a shift operator for the momentum also reflects itself in the commutation relations

$$
\left[\hat{p}_{R}, \widetilde{V}_{\pi / L}\right]=\frac{\pi}{L} \widetilde{V}_{\pi / L}, \quad\left[\hat{p}_{R}, \widetilde{V}_{\pi / L}^{\dagger}\right]=-\frac{\pi}{L} \widetilde{V}_{\pi / L}^{\dagger} .
$$


Since for $x=0$ and $x=L$ the operator $\widetilde{V}_{\pi / L}$ reduces to $\mathbb{1}$, for a wave function $\Psi(x) \in D\left(\hat{p}_{R}\right)$ [which obeys $\Psi_{o}(0)=$ $\lambda \Psi_{e}(0)$ and $\left.\Psi_{o}(L)=\lambda_{L} \Psi_{e}(L)\right]$ one obtains $\left(\widetilde{V}_{\pi / L} \Psi\right)_{o}(0)=$ $\lambda\left(\widetilde{V}_{\pi / L} \Psi\right)_{e}(0)$ and $\left(\widetilde{V}_{\pi / L} \Psi\right)_{o}(L)=\lambda_{L}\left(\tilde{V}_{\pi / L} \Psi\right)_{e}(L)$. As a result, $\widetilde{V}_{\pi / L} \Psi(x) \in D\left(\hat{p}_{R}\right)$, which means that $\widetilde{V}_{\pi / L}$ does not lead out of the domain $D\left(\hat{p}_{R}\right)$. This implies that the commutation relations of Eq. (65) are not just formal relations, but are fully consistent with the domains of the corresponding operators.

It should be noted that the canonical commutation relation $\left[\hat{x}_{R}, \hat{p}_{R}\right]=i$, which is completely appropriate on the halfline, is no longer a meaningful expression in the interval. This is because, in contrast to $\widetilde{V}_{\pi / L}$, the operator $\hat{x}_{R}$ leads out of the domain $D\left(\hat{p}_{R}\right)$. This follows from $\left(\hat{x}_{R} \Psi\right)_{e}(L)=$ $L \Psi_{o}(L)=L \lambda_{L} \Psi_{e}(L)=\lambda_{L}\left(\hat{x}_{R} \Psi\right)_{o}(L)$, which is inconsistent with the domain condition $\left(\hat{x}_{R} \Psi\right)_{o}(L)=\lambda_{L}\left(\hat{x}_{R} \Psi\right)_{o}(L)$. Hence, in the interval $[0, L]$ the commutation relations of Eq. (65) replace the canonical commutation relation $\left[\hat{x}_{R}, \hat{p}_{R}\right]=i$. This is another example where the last step in the canonical quantization procedure is crucial: "If the canonical commutation relation between position and momentum itself is compromised by domain incompatibilities, try to replace the position or the momentum operator by more appropriate operators that are unaffected by such subtleties." Starting from the original position and momentum operators $\hat{x}$ and $\hat{p}=-i \partial_{x}$, which no longer obey the canonical commutation relation on the halfline, we were led to the operators $\hat{x}_{R}=\sigma_{1} x$ and $\hat{p}_{R}=-i \sigma_{1} \partial_{x}$, which indeed satisfy $\left[\hat{x}_{R}, \hat{p}_{R}\right]=i$. Moving on to an interval, we now realize that this relation again seizes to hold, which motivated us to replace $\hat{x}_{R}$ by the more appropriate operator $\widetilde{V}_{\pi / L}=\exp \left(i \frac{\pi}{L} \hat{x}_{R}\right)$ that obeys the commutation relations Eq. (65).

How does the operator $V_{a}=\exp \left(i \hat{p}_{R} a\right)$ act on the $\hat{x}_{R}$ eigenstates $|x, \pm\rangle$ (with $x \in[0, L]$ ) of Eq. (48)? In close analogy to Eq. (50), for $a \in[-L, L)$ one obtains

$$
\begin{aligned}
& V_{a}|x,+\rangle=\sigma_{L}|a-x+2 L,-\rangle, \text { for } \mathrm{L} \leqslant \mathrm{x}-\mathrm{a}<2 \mathrm{~L}, \\
& V_{a}|x,+\rangle=|x-a,+\rangle, \text { for } 0 \leqslant \mathrm{x}-\mathrm{a}<\mathrm{L}, \\
& V_{a}|x,+\rangle=\sigma|a-x,-\rangle, \text { for }-\mathrm{L} \leqslant \mathrm{x}-\mathrm{a}<0, \\
& V_{a}|x,-\rangle=\sigma_{L}^{*}|-x-a+2 L,+\rangle, \text { for } \mathrm{L} \leqslant \mathrm{x}+\mathrm{a}<2 \mathrm{~L}, \\
& V_{a}|x,-\rangle=|x+a,-\rangle, \text { for } 0 \leqslant \mathrm{x}+\mathrm{a}<\mathrm{L}, \\
& V_{a}|x,-\rangle=\sigma^{*}|-x-a,+\rangle, \text { for }-\mathrm{L} \leqslant \mathrm{x}+\mathrm{a}<0 .
\end{aligned}
$$

Independently of $\sigma$ or $\sigma_{L}$, as a consequence of Eq. (65) one then obtains the Weyl group relation

$$
V_{a} \widetilde{V}_{\pi / L}=\exp \left(i \frac{\pi}{L} a\right) \widetilde{V}_{\pi / L} V_{a} .
$$

The action of the operators $V_{a}$ and $\widetilde{V}_{\pi / L}$ is illustrated in Fig. 3. Since in the interval momentum is quantized, in the corresponding momentum space there are only discrete translations by multiples of $\frac{\pi}{L}$, which are generated by $\widetilde{V}_{\pi / L}$. Despite the fact that there is no translation symmetry in the interval $[0, L]$ (which is the reason why the original momentum operator $\hat{p}=-i \partial_{x}$ is not self-adjoint), the momentum operator $\hat{p}_{R}=-i \sigma_{1} \partial_{x}$ indeed generates infinitesimal translations in the doubly covered interval $[0, L]$. As illustrated in Fig. 3, $V_{a}=\exp \left(i \hat{p}_{R} a\right)$ acts like a periodic "conveyor belt," which transports states $|x,+\rangle$ (in the finite-energy sector) to the left,

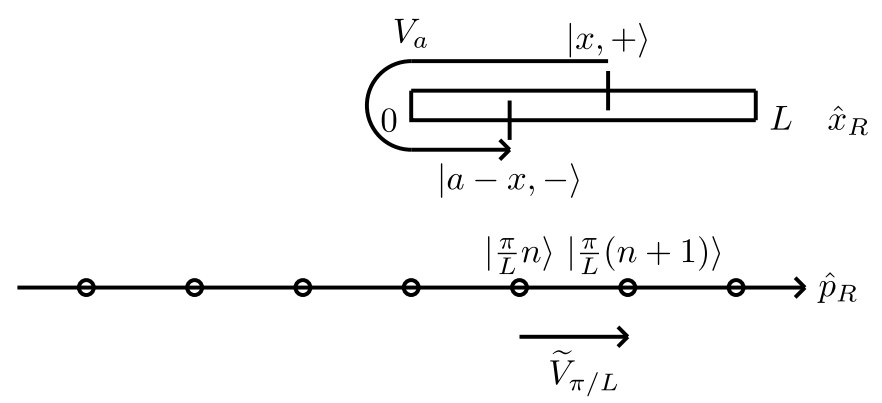

FIG. 3. Action of the translation operators $V_{a}=\exp \left(i \hat{p}_{R} a\right)$ and $\widetilde{V}_{\pi / L}=\exp \left(i \frac{\pi}{L} \hat{x}_{R}\right)$ in the continuous position and discrete momentum eigenstates for the interval $[0, L]$.

and states $|x,-\rangle$ (which lie in the sector of states whose energy is ultraviolet-sensitive) to the right. When a state $|x,+\rangle$ is transported beyond the origin (at $x=0$ ), it turns into the state $|a-x,-\rangle$. When such a state is transported further beyond the other end of the interval (at $x=L$ ), it returns to the finite-energy sector.

It is a matter of definition whether one wants to classify the situation in the interval as belonging to "canonical quantization." We prefer to do so, despite the fact that $\hat{x}_{R}$ and $\hat{p}_{R}$ no longer satisfy a meaningful canonical commutation relation. The reason for this is simply that in a finite volume momentum is quantized. As a consequence, $\hat{x}_{R}$ can no longer generate infinitesimal translations in momentum space. The appropriate discrete momentum translations by $\frac{\pi}{L}$ are generated by $\widetilde{V}_{\pi / L}=\exp \left(i \frac{\pi}{L} \hat{x}_{R}\right)$. As a result, the commutation relations of Eq. (65) replace the usual canonical commutation relation. In any case, the corresponding Weyl group of Eq. (67) has the same form as in the other cases of canonical quantization, and thus using the term "canonical quantization" also for the interval is indeed justified.

\section{B. Comparison with motion on $S^{\mathbf{1}}$}

Let us compare the situation in an interval with the wellunderstood motion of a quantum mechanical particle on a circle $S^{1}$, parametrized by the angle $\varphi \in[-\pi, \pi]$. In that case, the operator for the linear momentum is replaced by the angular momentum operator $\hat{L}=-i \partial_{\varphi}$. First, let us investigate the Hermiticity and self-adjointness of $\hat{L}$, which acts on wave functions $\Psi(\varphi)$. Applying partial integration one obtains

$$
\begin{aligned}
& \left\langle\hat{L}^{\dagger} \chi \mid \Psi\right\rangle=\langle\chi \mid \hat{L} \Psi\rangle= \\
& \langle\hat{L} \chi \mid \Psi\rangle-i\left[\chi(\pi)^{*} \Psi(\pi)-\chi(-\pi)^{*} \Psi(-\pi)\right] .
\end{aligned}
$$

Hermiticity of $\hat{L}$ hence requires that the expression in square brackets vanishes. Since, in contrast to an open interval, $\varphi=$ $\pi$ and $\varphi=-\pi$ parametrize the same point on the closed circle, the boundary condition

$$
\Psi(\pi)=\rho \Psi(-\pi)
$$

which restricts the domain $D(\hat{L})$, is local and thus physically admissible. Inserting this relation in the square brackets in Eq. (68), and using the fact that $\Psi(-\pi)$ can still take arbitrary 
values, one obtains the Hermiticity condition

$$
\left[\chi(\pi)^{*} \rho-\chi(-\pi)^{*}\right] \Psi(-\pi)=0 \Rightarrow \chi(\pi)=\frac{1}{\rho^{*}} \chi(-\pi),
$$

which defines the domain $D\left(\hat{L}^{\dagger}\right)$. The operator $\hat{L}$ is selfadjoint only if $D(\hat{L})=D\left(\hat{L}^{\dagger}\right)$, which is the case when

$$
\rho=\frac{1}{\rho^{*}} \Rightarrow \rho=\exp (i \theta) \text {. }
$$

It is well known that the angular momentum operator $\hat{L}$ has a 1-parameter family of self-adjoint extensions parametrized by the angle $\theta$. The angular momentum eigenstates $|n\rangle$ then obey

$$
\begin{aligned}
& \hat{L}|n\rangle=\left(n+\frac{\theta}{2 \pi}\right)|n\rangle, \quad n \in \mathbb{Z}, \\
& \langle\varphi \mid n\rangle=\frac{1}{\sqrt{2 \pi}} \exp \left[i\left(n+\frac{\theta}{2 \pi}\right) \varphi\right], \\
& \langle\pi \mid n\rangle=\exp (i \theta)\langle-\pi \mid n\rangle .
\end{aligned}
$$

It is also well known that $\hat{\varphi}$ and $\hat{L}$ do not obey the standard canonical commutation relation. This is because an operation with the nonperiodic operator $\hat{\varphi}$ leads out of the domain of $D(\hat{L})$. Instead, the periodic unitary operator $\widetilde{U}=\exp (i \hat{\varphi})$ acts as a discrete translation operator of angular momentum, with which it obeys the commutation relations

$$
[\hat{L}, \tilde{U}]=\tilde{U}, \quad\left[\hat{L}, \widetilde{U}^{\dagger}\right]=-\widetilde{U}^{\dagger} .
$$

Defining angular eigenstates $|\varphi\rangle$ by $\hat{\varphi}|\varphi\rangle=\varphi|\varphi\rangle$, the operator $U_{\alpha}=\exp (i \hat{L} \alpha)$, with $\alpha \in[-\pi, \pi)$, acts as

$$
\begin{aligned}
& U_{\alpha}|\varphi\rangle=\exp (i \theta)|\varphi-\alpha+2 \pi\rangle, \text { for }-2 \pi \leqslant \varphi-\alpha<-\pi, \\
& U_{\alpha}|\varphi\rangle=|\varphi-\alpha\rangle, \text { for }-\pi \leqslant \varphi-\alpha<\pi, \\
& U_{\alpha}|\varphi\rangle=\exp (-i \theta)|\varphi-\alpha-2 \pi\rangle, \text { for } \pi \leqslant \varphi-\alpha<2 \pi .
\end{aligned}
$$

As a result of Eq. (73), one then obtains the Weyl group relation

$$
U_{\alpha} \widetilde{U}=\exp (i \alpha) \widetilde{U} U_{\alpha} .
$$

The action of the operators $U_{\alpha}$ and $\widetilde{U}$ is illustrated in Fig. 4. Angular momentum is quantized in integer units (shifted by $\left.\frac{\theta}{2 \pi}\right)$, and the corresponding discrete translation symmetry is generated by $\widetilde{U}$. The continuous angular rotations described by $U_{\alpha}=\exp (i \hat{L} \alpha)$ act like the periodic "conveyor belt" associated with $V_{a}=\exp \left(i \hat{p}_{R} a\right)$ for the interval that is illustrated in Fig. 3.

The similarities between Eq. (73) for the circle $S^{1}=$ $[-\pi, \pi]$ and Eq. (65) for the interval $[0, L]$ are not accidental. In fact, the operator pairs $\hat{L}, \widetilde{V}$ and $\hat{p}_{R}, \widetilde{V}_{\pi / L}$ are mathematically related by a unitary transformation, although they are physically quite different. Up to phase factors, the unitary transformation $U$ maps the position eigenstates $|x, \pm\rangle$ in the interval $x \in[0, L]$ to the angular eigenstates $|\varphi\rangle$ on the circle $\varphi \in[-\pi, \pi]:$

$$
\begin{aligned}
U V_{a} U^{\dagger} & =U_{\alpha=\pi a / L}, \quad U \widetilde{V}_{\pi / L} U^{\dagger}=\widetilde{U}, \\
U \hat{x}_{R} U^{\dagger} & =\frac{L}{\pi} \hat{\varphi},
\end{aligned}
$$

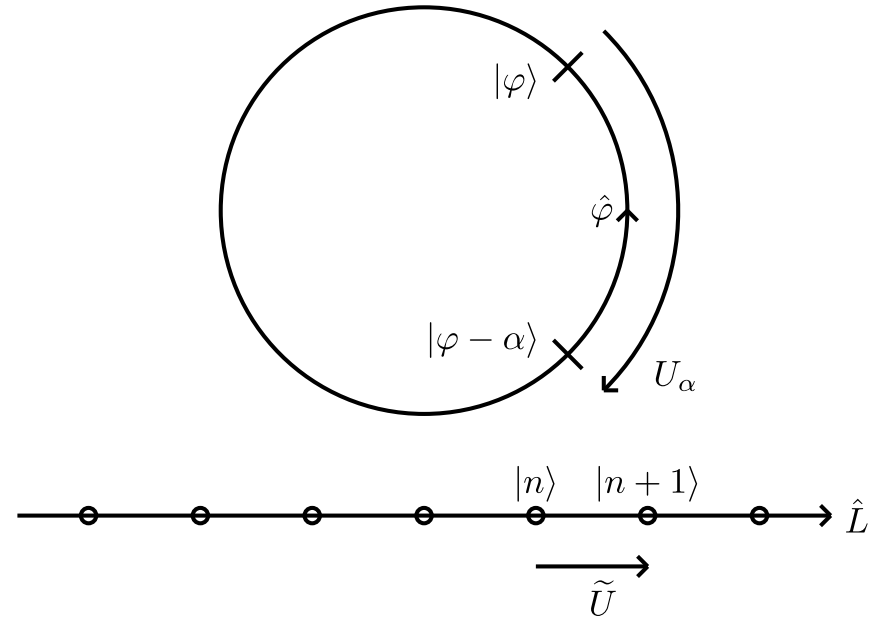

FIG. 4. Action of the translation operators $U_{\alpha}=\exp (i \hat{L} \alpha)$ and $\widetilde{U}=\exp (i \hat{\varphi})$ on the continuous angle and discrete angular momentum eigenstates for the circle $S^{1}=[-\pi, \pi]$.

$$
\begin{aligned}
& U|x,+\rangle=\left|\varphi=\frac{\pi x}{L}\right\rangle, \quad U|x,-\rangle=\sigma^{*}\left|\varphi=-\frac{\pi x}{L}\right\rangle, \\
& U \hat{p}_{R} U^{\dagger}=\frac{\pi}{L} \hat{L}, \quad U\left|\phi_{k}\right\rangle=\left|n=\frac{k L}{\pi}\right\rangle .
\end{aligned}
$$

Let us check this for consistency. For example, using Eq. (66) for $L \leqslant x-a<2 L$, which implies $\pi \leqslant \varphi-\alpha<2 \pi$, we obtain

$$
\begin{aligned}
& V_{a}|x,+\rangle=\sigma_{L}|a-x+2 L,-\rangle \Rightarrow \\
& U V_{a} U^{\dagger} U|x,+\rangle=\sigma_{L} U|a-x+2 L,-\rangle \Rightarrow \\
& U_{\alpha=\pi a / L}\left|\varphi=\frac{\pi x}{L}\right\rangle=\sigma_{L} \sigma^{*}\left|\varphi=-\frac{\pi}{L}(a-x+2 L)\right\rangle \Rightarrow \\
& \exp (-i \theta)\left|\varphi=\frac{\pi}{L}(x-a)-2 \pi\right\rangle \\
& =\sigma_{L} \sigma^{*}\left|\varphi=\frac{\pi}{L}(x-a)-2 \pi\right\rangle .
\end{aligned}
$$

This is indeed consistent, because based on Eq. (61), $\exp (i \theta)=\sigma \sigma_{L}^{*}$.

Equation (76) is very similar to Eq. (52) which describes the mathematical unitary equivalence of the standard operators $\hat{x}$ and $\hat{p}$ acting on wave functions over the entire real axis with the operators $\hat{x}_{R}$ and $\hat{p}_{R}$ that apply on the half-line, that followed from the Stone-von Neumann theorem. Also in that case the mathematical unitary equivalence relates two physically very different situations.

\section{Physical significance of $\theta$}

For circular motion, the parameter $\theta$ is well known to represent a magnetic flux that threads the circle, which affects a charged particle moving around the circle via an AharonovBohm phase. First of all, since the angles $\varphi= \pm \pi$ parametrize one and the same point on the circle, in the absence of magnetic flux the value of the wave function should be unique at that point, i.e., $\Psi(\pi)=\Psi(-\pi)$, such that $\theta=0$. In the presence of a magnetic flux $\Phi$, the angular momentum operator 
takes the form

$$
\hat{L}^{\prime}=-i \partial_{\varphi}+\frac{e \Phi}{2 \pi}=-i \partial_{\varphi}+\frac{\theta}{2 \pi}, \quad \theta=e \Phi .
$$

Here $-e$ is the charge of the particle and (along with $\hbar$ ) we have put the velocity of light to 1 . One can now perform the unitary transformation

$$
\begin{aligned}
& U(\theta)=\exp \left(i \frac{\theta}{2 \pi} \hat{\varphi}\right), \\
& \hat{L}=U(\theta) \hat{L}^{\prime} U(\theta)^{\dagger}=-i \partial_{\varphi}, \Psi(\varphi)=U(\theta) \Psi^{\prime}(\varphi),
\end{aligned}
$$

which implies

$$
\begin{aligned}
\Psi(\pi) & =\exp \left(i \frac{\theta}{2}\right) \Psi^{\prime}(\pi)=\exp \left(i \frac{\theta}{2}\right) \Psi^{\prime}(-\pi) \\
& =\exp (i \theta) \Psi(-\pi) .
\end{aligned}
$$

After the unitary transformation, the wave function $\Psi(\varphi)$ is no longer single-valued at $\varphi= \pm \pi$. This is no problem, because the unitary transformation corresponds to a nonperiodic gauge transformation. Like the magnetic flux $\Phi$, the parameter $\theta$ itself is gauge invariant. However, the unitary transformation $U(\theta)$ moves $\theta$ from the operator $\hat{L}^{\prime}$ to the (no longer strictly periodic) wave function $\Psi(\varphi)$.

The similarity with the situation in the interval suggests that a similar interpretation exists for the corresponding parameter $\theta$, which results from

$$
\exp (i \theta)=\sigma \sigma_{L}^{*}=\frac{(1-\lambda)\left(1+\lambda_{L}\right)}{(1+\lambda)\left(1-\lambda_{L}\right)} .
$$

As we have seen, the unitary transformation $W=\exp \left(i \omega \sigma_{1}\right)$ of Eq. (45) changes the values of the self-adjoint extension parameters, such that $\sigma^{\prime}=\exp (-2 i \omega) \sigma, \sigma_{L}^{\prime}=\exp (-2 i \omega) \sigma_{L}$, but leaves $\theta$ invariant [cf. Eq. (63)]. Still, we can perform another $x$-dependent unitary transformation,

$$
W(\theta)=\exp \left(i \frac{\theta}{2 L} \sigma_{1} \hat{x}\right)=\exp \left(i \frac{\theta}{2 L} \hat{x}_{R}\right),
$$

which implies $\sigma^{\prime}=\sigma$ and $\sigma_{L}^{\prime}=\exp (-i \theta) \sigma_{L}$ such that $\sigma^{\prime} \sigma_{L}^{\prime *}=1$. The transformation $W(\theta)$ again represents a gauge transformation in Hilbert space, which now moves $\theta$ from the boundary conditions on the wave function to the momentum operator

$$
\hat{p}_{R}^{\prime}=W(\theta)^{\dagger} \hat{p}_{R} W(\theta)=-i \sigma_{1} \partial_{x}+\frac{\theta}{2 L} .
$$

When we perform the unitary transformation on the kinetic energy $\hat{T}$ we obtain

$$
\begin{aligned}
& \hat{T}^{\prime}=W(\theta)^{\dagger} \hat{T} W(\theta)=\frac{\hat{p}^{\prime 2}}{2 m} \mathbb{1}, \\
& \hat{p}^{\prime}=-i \partial_{x}+\frac{\theta}{2 L}=\hat{p}+e A_{x}, \quad \theta=2 e A_{x} L .
\end{aligned}
$$

In this case, $\theta$ manifests itself as a vector potential $A_{x}$, or more precisely as its line integral $\int_{0}^{L} d x A_{x}=A_{x} L$ that connects the two boundaries. The original Robin boundary condition, $\gamma \Psi(0)-\partial_{x} \Psi(0)=0$, of Eq. (3), along with its counterpart at the other end of the interval, $\gamma_{L} \Psi(L)+\partial_{x} \Psi(L)=0$, must also be transformed accordingly, and one obtains [35]

$$
\begin{aligned}
& \gamma \Psi^{\prime}(0)-D_{x} \Psi^{\prime}(0)=0, \gamma_{L} \Psi^{\prime}(L)+D_{x} \Psi^{\prime}(L)=0, \\
& D_{x}=\partial_{x}+i e A_{x} .
\end{aligned}
$$

The covariant derivative $D_{x}$ also enters the conserved probability current

$$
j^{\prime}(x)=\frac{1}{2 m i}\left[\Psi^{\prime}(x)^{*} D_{x} \Psi^{\prime}(x)-D_{x} \Psi^{\prime}(x)^{*} \Psi^{\prime}(x)\right] .
$$

As a result, the boundary conditions of Eq. (85) still guarantee that no probability leaks out of the interval, i.e., $j^{\prime}(0)=$ $j^{\prime}(L)=0$.

Under a general gauge transformation $\varphi(x)$ the vector potential and the wave function transform as

$$
{ }^{\varphi} A_{x}(x)=A_{x}(x)-\partial_{x} \varphi(x), \quad{ }^{\varphi} \Psi^{\prime}(x)=\exp [\operatorname{ie} \varphi(x)] \Psi^{\prime}(x) .
$$

The gauge string connecting the two ends of the interval, capped by the values of the wave function at the end points,

$$
\begin{aligned}
S & =|0\rangle \exp \left(\text { ie } \int_{0}^{L} d x A_{x}(x)\right)\langle L|, \\
\left\langle\Psi^{\prime}|S| \Psi^{\prime}\right\rangle & =\Psi^{\prime}(0)^{*} \exp \left(\text { ie } \int_{0}^{L} d x A_{x}(x)\right) \Psi^{\prime}(L),
\end{aligned}
$$

is gauge invariant, i.e., $\left\langle\left.\left.{ }^{\varphi} \Psi^{\prime}\right|^{\varphi} S\right|^{\varphi} \Psi^{\prime}\right\rangle=\left\langle\Psi^{\prime}|S| \Psi^{\prime}\right\rangle$. In cases where such a gauge string stretches through the interval, the parameter $\theta$ appears in the corresponding momentum operator.

\section{Momentum measurements in $[0, L]$}

Let us now consider momentum measurements in the interval $[0, L]$. For simplicity, we consider a Hamiltonian without a potential [i.e., $V(x)=0$ ]. First, we investigate Neumann boundary conditions, which are characterized by $\gamma=0$ at both ends of the interval. In the limit $\mu \rightarrow \infty$, the finiteenergy eigenstates of $\hat{H}(\mu)$ then take the form

$$
\begin{aligned}
\hat{H}(\mu) \psi_{l}(x) & =E_{l} \psi_{l}(x), \quad E_{l}=\frac{\pi^{2} l^{2}}{2 m L^{2}}, \quad l \in \mathbb{N}_{\geqslant 0}, \\
\psi_{0}(x) & =\frac{1}{\sqrt{2 L}}\left(\begin{array}{l}
1 \\
1
\end{array}\right), \\
\psi_{l>0}(x) & =\frac{1}{\sqrt{L}}\left(\begin{array}{l}
\cos (\pi l x / L) \\
\cos (\pi l x / L)
\end{array}\right) .
\end{aligned}
$$

Again for simplicity, we choose $\lambda_{L}=\lambda$ which implies $\theta=$ 0 , such that the corresponding momentum eigenvalues and eigenfunctions are

$$
\begin{aligned}
\hat{p}_{R} \phi_{k}(x) & =k \phi_{k}(x), k=\frac{\pi n}{L}, n \in \mathbb{Z}, \sigma=\frac{1-\lambda}{1+\lambda} \in U(1), \\
\phi_{k}(x) & =\frac{1}{2 \sqrt{L}}\left(\begin{array}{l}
\exp (i k x)+\sigma \exp (-i k x) \\
\exp (i k x)-\sigma \exp (-i k x)
\end{array}\right) .
\end{aligned}
$$

First of all, one obtains $\left\langle\psi_{l}\left|\hat{p}_{R}\right| \psi_{l}\right\rangle=0$. Irrespective of the value of $\lambda$, when one projects them onto the finite-energy sector, the momentum eigenstates are just $\phi_{k, e}^{+}(x)=\phi_{k, o}^{+}(x)=$ $\frac{1}{2 \sqrt{L}} \exp (i k x)$. In the ground state the probability to measure the momentum value $k=0$ is $\left|\left\langle\phi_{0} \mid \psi_{0}\right\rangle\right|^{2}=\frac{1}{2}$. Similarly, the 


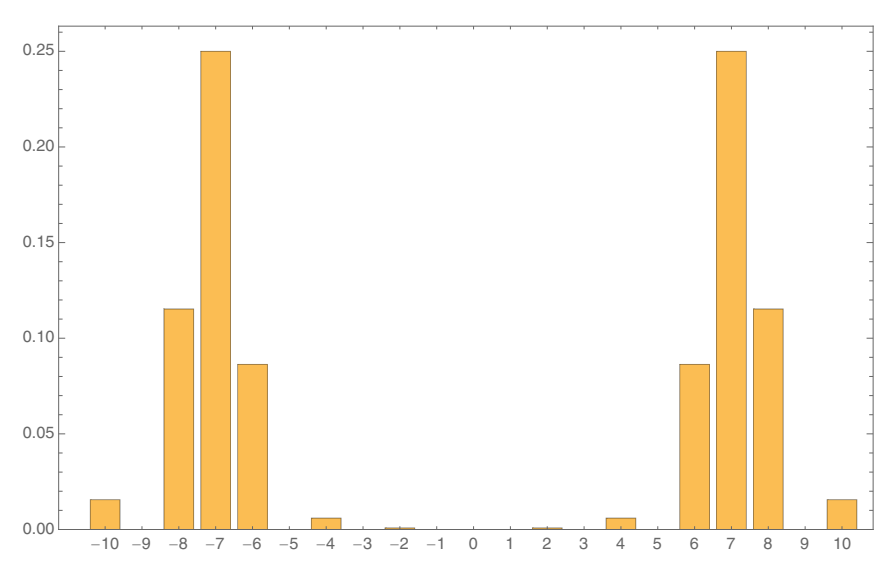

FIG. 5. Probability to measure the momentum $k=\frac{\pi}{L} n$ in the energy eigenstate $\psi_{l}(x)$ with $l=7$ for Neumann boundary conditions, as a function of $n \in\{-10, \ldots, 10\}$.

probability to measure $k=\frac{\pi n}{L}$ with $n \neq 0$ is

$$
\left|\left\langle\phi_{k} \mid \psi_{0}\right\rangle\right|^{2}=\frac{2}{\pi^{2} n^{2}},
$$

for odd $n$ and zero otherwise. When one measures the momentum in an energy eigenstate $\psi_{l}(x)$ with $l>0$, one obtains $k= \pm \frac{\pi l}{L}$ each with probability $\frac{1}{4}$. The probability to measure $k=\frac{\pi n}{L}$ for $n \neq \pm l$ is then given by

$$
\left|\left\langle\phi_{k} \mid \psi_{l}\right\rangle\right|^{2}=\frac{4 n^{2}}{\pi^{2}\left(l^{2}-n^{2}\right)^{2}},
$$

if $(-1)^{n}=-(-1)^{l}$ and zero otherwise. Indeed the various probabilities, which are illustrated in Fig. 5, are correctly normalized because

$$
\begin{aligned}
& \frac{1}{2}+\sum_{n \in \mathbb{Z}, n \text { odd }} \frac{2}{\pi^{2} n^{2}}=1, \text { for } l=0, \\
& \frac{1}{4}+\frac{1}{4}+\sum_{n \in \mathbb{Z}, n \text { even }} \frac{4 n^{2}}{\pi^{2}\left(l^{2}-n^{2}\right)^{2}}=1, \text { for odd } l>0, \\
& \frac{1}{4}+\frac{1}{4}+\sum_{n \in \mathbb{Z}, n \text { odd }} \frac{4 n^{2}}{\pi^{2}\left(l^{2}-n^{2}\right)^{2}}=1, \text { for even } l>0 .
\end{aligned}
$$

Let us also consider the momentum uncertainty $\left(\Delta p_{R}\right)^{2}=$ $\left\langle\hat{p}_{R}^{2}\right\rangle-\left\langle\hat{p}_{R}\right\rangle^{2}$ in the energy eigenstate $\psi_{l}(x)$. Besides $\left\langle\hat{p}_{R}\right\rangle=0$ one obtains

$$
\begin{aligned}
\left\langle\hat{p}_{R}^{2}\right\rangle & =\frac{\pi^{2}}{L^{2}} \sum_{n \in \mathbb{Z}, n \text { odd }} \frac{2}{\pi^{2}} \rightarrow \infty, \text { for } l=0, \\
\left\langle\hat{p}_{R}^{2}\right\rangle & =\frac{\pi^{2}}{L^{2}}\left(\frac{l^{2}}{4}+\frac{(-l)^{2}}{4}+\sum_{n \in \mathbb{Z}, n \text { even }} \frac{4 n^{4}}{\pi^{2}\left(l^{2}-n^{2}\right)^{2}}\right) \\
& \rightarrow \infty, \text { for odd } l>0, \\
\left\langle\hat{p}_{R}^{2}\right\rangle & =\frac{\pi^{2}}{L^{2}}\left(\frac{l^{2}}{4}+\frac{(-l)^{2}}{4}+\sum_{n \in \mathbb{Z}, n \text { odd }} \frac{4 n^{4}}{\pi^{2}\left(l^{2}-n^{2}\right)^{2}}\right) \\
& \rightarrow \infty, \text { for even } l>0 .
\end{aligned}
$$

Hence, for Neumann boundary conditions the momentum uncertainty diverges for any energy eigenstate. This is a consequence of the domain incompatibility $D(\hat{T}) \neq D\left(\hat{p}_{R}\right)$. Although at a formal level of differential expressions the kinetic energy operator $\hat{T}$ and the momentum operator $\hat{p}_{R}$ seem to commute (which would imply a vanishing momentum uncertainty), the domain incompatibility leads to a completely different result.

Let us now consider the limit $L \rightarrow \infty$ in which we expect to recover the results for the half-line. For $\gamma=0$, according to Eq. (6), $R(p)=1$. Then, using Eq. (19), for $k \neq \pm p$ one obtains

$$
\left|\left\langle k \mid \psi_{E}\right\rangle\right|^{2}=\frac{4 k^{2}}{\left(p^{2}-k^{2}\right)^{2}}, \quad E=\frac{p^{2}}{2 m} .
$$

For finite $L$ we identify $k=\frac{\pi n}{L}$ and $p=\frac{\pi l}{L}$, such that, using Eq. (92) for $n \neq \pm l$, one gets

$$
\begin{aligned}
\left|\left\langle\phi_{k} \mid \psi_{l}\right\rangle\right|^{2} & =\frac{4 n^{2}}{\pi^{2}\left(l^{2}-n^{2}\right)^{2}}=\frac{1}{L^{2}} \frac{4 k^{2}}{\left(p^{2}-k^{2}\right)^{2}} \\
& =\frac{1}{L^{2}}\left|\left\langle k \mid \psi_{E}\right\rangle\right|^{2} .
\end{aligned}
$$

The factor $\frac{1}{L^{2}}$ is due to the fact that $|k\rangle$ and $\left|\Psi_{E}\right\rangle$ are normalized to $\delta$ functions, while $\left|\phi_{k}\right\rangle$ and $\left|\psi_{l}\right\rangle$ are normalized to 1 . In any case, this implies that (with Neumann boundary conditions) in the stationary scattering state $\psi_{E}(x)=\exp (-i p x)+$ $\exp (i p x)$ the probability to measure a momentum value $k=p$ or $k=-p$ is $\frac{1}{4}$ in both cases. In the remaining half of the cases the momentum measurement returns a result $k \neq \pm p$ with a divergent momentum uncertainty. This somewhat counterintuitive result is again due to domain incompatibilities.

Finally, let us also discuss the standard textbook case of Dirichlet boundary conditions which are characterized by $\gamma=$ $\infty$. The spectrum of finite-energy states then takes the form

$$
\begin{aligned}
\hat{H}(\mu) \psi_{l}(x) & =E_{l} \psi_{l}(x), \quad E_{l}=\frac{\pi^{2} l^{2}}{2 m L^{2}}, \quad l \in \mathbb{N}_{>0}, \\
\psi_{l}(x) & =\frac{1}{\sqrt{L}}\left(\begin{array}{c}
\sin (\pi l x / L) \\
\sin (\pi l x / L)
\end{array}\right) .
\end{aligned}
$$

In this case, the probability to measure the momentum $k=$ $p$ or $k=-p$ is again $\frac{1}{4}$ in both cases, and the probability to measure $k \neq \pm p$ is

$$
\left|\left\langle\phi_{k} \mid \psi_{l}\right\rangle\right|^{2}=\frac{4 l^{2}}{\pi^{2}\left(l^{2}-n^{2}\right)^{2}},
$$

if $(-1)^{n}=-(-1)^{l}$ and zero otherwise. Again, the probabilities, which are illustrated in Fig. 6, are correctly normalized because

$$
\begin{aligned}
& \frac{1}{4}+\frac{1}{4}+\sum_{n \in \mathbb{Z}, n \text { even }} \frac{4 l^{2}}{\pi^{2}\left(l^{2}-n^{2}\right)^{2}}=1, \text { for odd } l>0, \\
& \frac{1}{4}+\frac{1}{4}+\sum_{n \in \mathbb{Z}, n \text { odd }} \frac{4 l^{2}}{\pi^{2}\left(l^{2}-n^{2}\right)^{2}}=1, \text { for even } l>0 .
\end{aligned}
$$




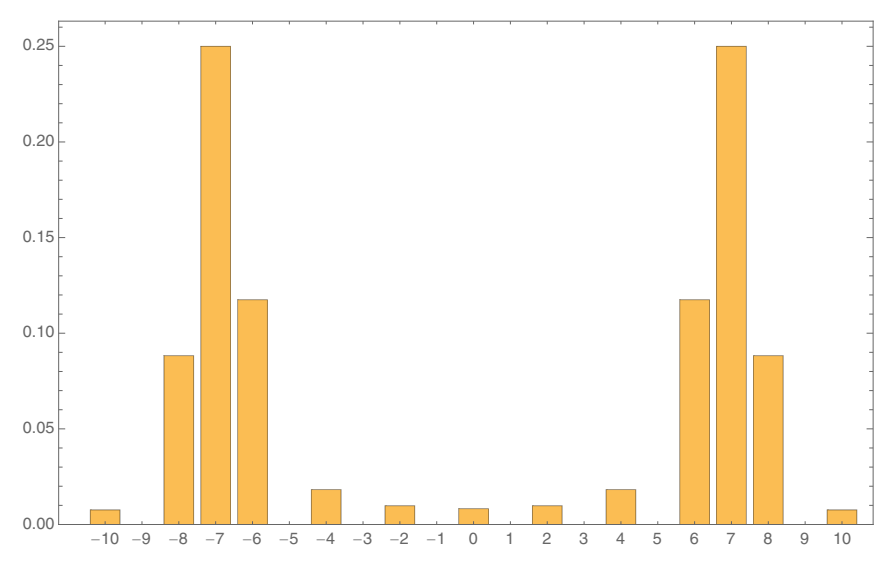

FIG. 6. Probability to measure the momentum $k=\frac{\pi}{L} n$ in the energy eigenstate $\psi_{l}(x)$ with $l=7$ for Dirichlet boundary conditions, as a function of $n \in\{-10, \ldots, 10\}$.

In this case one obtains

$$
\begin{aligned}
\left\langle\hat{p}_{R}^{2}\right\rangle & =\frac{\pi^{2}}{L^{2}}\left(\frac{l^{2}}{4}+\frac{(-l)^{2}}{4}+\sum_{n \in \mathbb{Z}, n \text { even }} \frac{4 l^{2} n^{2}}{\pi^{2}\left(l^{2}-n^{2}\right)^{2}}\right) \\
& =\frac{\pi^{2} l^{2}}{L^{2}}, \text { for odd } l>0, \\
\left\langle\hat{p}_{R}^{2}\right\rangle & =\frac{\pi^{2}}{L^{2}}\left(\frac{l^{2}}{4}+\frac{(-l)^{2}}{4}+\sum_{n \in \mathbb{Z}, n \text { odd }} \frac{4 l^{2} n^{2}}{\pi^{2}\left(l^{2}-n^{2}\right)^{2}}\right) \\
& =\frac{\pi^{2} l^{2}}{L^{2}}, \text { for even } l>0,
\end{aligned}
$$

which implies $\left(\Delta p_{R}\right)^{2}=2 m E_{l}$. Hence, for Dirichlet boundary conditions the momentum uncertainty in an energy eigenstate is finite. This is because, in this case, $D(\hat{T}) \subset D\left(\hat{p}_{R}\right)$. Again using Eq. (6), one now gets $R(p)=-1$, and following Eq. (19), for $k \neq \pm p$ one obtains

$$
\left|\left\langle k \mid \psi_{E}\right\rangle\right|^{2}=\frac{4 p^{2}}{\left(p^{2}-k^{2}\right)^{2}}=L^{2}\left|\left\langle\phi_{k} \mid \psi_{l}\right\rangle\right|^{2} .
$$

For Dirichlet boundary conditions the stationary scattering state is $\psi_{E}(x)=\exp (-i p x)-\exp (i p x)$. Then the probability to measure momentum $k=p$ or $k=-p$ is again $\frac{1}{4}$ in both cases. In the remaining half of the cases the momentum measurement results in $k \neq \pm p$, now with a finite momentum uncertainty.

\section{CONCLUSIONS}

We have introduced an alternative concept for a self-adjoint quantum mechanical momentum operator for an interval $[0, L]$ and for the half-line $\mathbb{R}_{\geqslant 0}$. The alternative concept arises naturally in the continuum limit of the lattice-regularized problem. On the lattice one distinguishes even and odd lattice points. In the continuum limit, this naturally leads to a two-component wave function, which is associated with a doubling of the Hilbert space from $L^{2}([0, L])$ to $L^{2}([0, L]) \times$ $\mathbb{C}^{2}$ and from $L^{2}\left(\mathbb{R}_{\geqslant 0}\right)$ to $L^{2}\left(\mathbb{R}_{\geqslant 0}\right) \times \mathbb{C}^{2}$. The additional continuum states correspond to lattice states with energies at the cutoff scale. In the continuum limit, these states are removed from the physical energy spectrum. The key insight underlying the alternative concept is that these states, although they are ultimately removed to infinite energy, must be kept in the physical description in order to facilitate the construction of a self-adjoint momentum operator. Interestingly, although the resulting momentum operator for the half-line is endowed with a self-adjoint extension parameter $\lambda$ (associated with the origin) and is thus not unique, the results of momentum measurements performed on finite-energy states are independent of this parameter. In an interval, the momentum operator is characterized by two self-adjoint extension parameters $\lambda$ and $\lambda_{L}$ (associated with the two boundary points). In that case, the value of the quantized momentum, $k=\frac{\pi}{L}\left(n+\frac{\theta}{2 \pi}\right)$, depends on the particular combination $\theta$ of the two self-adjoint extension parameters.

Based upon the alternative concept for the momentum operator, canonical quantization becomes applicable both to the half-line and to an interval. However, due to the existence of sharp boundaries, self-adjoint extension parameters enter the description and thus lead to physically inequivalent quantum variants of the same classical system. This goes beyond the usual operator ordering ambiguities. In particular, different operators, like the momentum $\hat{p}_{R}$ and the Hamiltonian $\hat{H}(\mu)$, act in different domains, $D\left(\hat{p}_{R}\right) \neq D(\hat{H}(\mu))$, of the Hilbert space. As a consequence, the commutation relations that result from classical Poisson bracket relations are just formal equations relating differential expressions. Understanding the true nature of the relations between the various operators requires a careful analysis of the corresponding operator domains.

This is an inevitable consequence of the low-energy continuum description of a system with sharp impenetrable boundaries, which are necessarily ultraviolet sensitive. Working explicitly with an ultraviolet lattice cutoff (representing the shortest physically relevant distance scale) is straightforward and might even be quite physical, but is not very transparent. A transparent effective low-energy continuum description, as it is completely common in quantum mechanics, necessarily uses an infinite-dimensional Hilbert space. As we have seen, on the half-line or in an interval this requires a careful application of the canonical quantization procedure. Familiarizing ourselves with the elegant mathematical framework originally established by von Neumann is very well worth the effort in order to gain a more complete physical understanding of these "simple" quantum systems.

It should be mentioned that most of the somewhat subtle Hilbert space and operator domain issues, associated with the canonical quantization procedure that we carried out above, arose only because we decided to construct the momentum operator in addition to just the Hamiltonian. To a physicist who favors Everett's many-worlds interpretation of quantum mechanics [47], this may seem unnecessary, because any measurement process is then incorporated in the global Hamiltonian that governs the time evolution of the wave function of the entire universe. This wave function includes the quantum system under study, any device that registers measurement results, as well as the conscious observer who reads off those results and uses them to draw conclusions about how the quantum system works. Still, when engaging in an actual study of an isolated quantum system (rather than of the universe as a whole), even a hard-line "Everettian" 


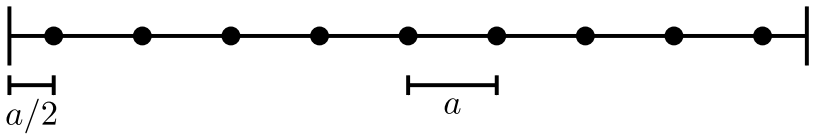

FIG. 7. Lattice with $N=9$ points in the interval $[0, L]$.

would probably prefer to work with the much more tractable canonical quantization procedure applied above.

The alternative concept of momentum in a space with boundaries has potentially far reaching consequences. Its generalization to higher dimensions is straightforward and was sketched in [9]. A natural next step, which is currently under investigation, concerns the generalization and physical interpretation of the Heisenberg uncertainty relation that was derived for a finite volume with sharp boundaries in [35]. This can be applied, for example, to optical box traps [1] and quantum dots [2], which may make the alternative momentum concept experimentally accessible. In this context, it is also interesting to construct a momentum measurement device, at least at a theoretical level, for example, along the lines originally introduced by von Neumann [27]. This implies coupling the quantum particle to another quantum system that serves as a measurement device, whose pointer can then be read out at the classical level. Time-of-flight momentum measurements of this kind have been discussed, for example, in [48]. Further applications, again along the lines of [35], suggest themselves in the context of relativistic fermions, for example, to the phenomenological MIT bag model [3-5], or to domain wall fermions residing in an interval of extra-dimensional space [6,7]. Canonical quantization (which was thought to be inapplicable because the standard momentum operator is not self-adjoint) can be applied in all these situations based on the alternative concept of momentum in a space with sharp boundaries.

\section{ACKNOWLEDGMENTS}

We would like to thank Immanuel Albrecht, Matthias Blau, Jan Herrmann, Christiane Tretter, and Valentin Wyss for illuminating discussions. The research of M.H.A. is supported by Madeleine Müller. U.-J.W. is supported by a grant of the Schweizerischer Nationalfonds.

\section{APPENDIX: LATTICE REGULARIZATION OF THE MOMENTUM OPERATOR}

In order to circumvent the subtleties associated with Hermiticity versus self-adjointness, which arise because the Hilbert space is infinite-dimensional, in [9] we have investigated the problem on the interval in an ultraviolet lattice regularization. As illustrated in Fig. 7, the interval $[0, L]$ is then divided into $N=L / a$ segments of size $a$ [not to be confused with the self-adjoint extension parameter of Eq. (38)], with a lattice point in the middle of each segment, such that $x=\left(n-\frac{1}{2}\right) a, n \in\{1,2, \ldots, N\}$. The Hilbert space then becomes $N$-dimensional and self-adjointness becomes indistinguishable from Hermiticity. As a result, there are no longer any domain issues, since every operator can act in the entire finite-dimensional Hilbert space.
The lattice momentum operator is represented by forward and backward discretized derivatives:

$$
\begin{gathered}
\hat{p}_{F}=-\frac{i}{a}\left(\begin{array}{ccccccc}
-1 & 1 & 0 & \ldots & 0 & 0 & 0 \\
0 & -1 & 1 & \ldots & 0 & 0 & 0 \\
0 & 0 & -1 & \ldots & 0 & 0 & 0 \\
& . & . & \ldots & . & . & . \\
& . & . & \ldots & . & . & . \\
0 & 0 & 0 & \ldots & -1 & 1 & 0 \\
0 & 0 & 0 & \ldots & 0 & -1 & 1 \\
0 & 0 & 0 & \ldots & 0 & 0 & \lambda_{L}
\end{array}\right), \\
\left.\hat{p}_{B}=-\frac{i}{a}\left(\begin{array}{ccccccc}
-\lambda & 0 & 0 & \ldots & 0 & 0 & 0 \\
-1 & 1 & 0 & \ldots & 0 & 0 & 0 \\
0 & -1 & 1 & \ldots & 0 & 0 & 0 \\
& . & . & \ldots & . & . & . \\
0 & . & . & \ldots & . & . & . \\
0 & 0 & 0 & \ldots & 1 & 0 & 0 \\
0 & 0 & 0 & \ldots & -1 & 1 & 0 \\
& 0 & 0 & \ldots & 0 & -1 & 1
\end{array}\right) . \text { (A }\right)
\end{gathered}
$$

On the lattice, the parameters $\lambda, \lambda_{L} \in i \mathbb{R}$ are directly incorporated in the corresponding matrices. In the continuum limit $a \rightarrow 0$ they turn into self-adjoint extension parameters. Neither $\hat{p}_{F}$ nor $\hat{p}_{B}$ are Hermitian matrices. It is natural to construct the following combinations:

$$
\begin{aligned}
& \hat{p}_{R}=\frac{1}{4}\left(\hat{p}_{F}+\hat{p}_{F}^{\dagger}+\hat{p}_{B}+\hat{p}_{B}^{\dagger}\right) \\
& =-\frac{i}{2 a}\left(\begin{array}{ccccccc}
-\lambda & 1 & 0 & \ldots & 0 & 0 & 0 \\
-1 & 0 & 1 & \ldots & 0 & 0 & 0 \\
0 & -1 & 0 & \ldots & 0 & 0 & 0 \\
& . & . & \ldots & . & . & . \\
& . & . & \ldots & . & . & . \\
0 & 0 & 0 & \ldots & 0 & 1 & 0 \\
0 & 0 & 0 & \ldots & -1 & 0 & 1 \\
0 & 0 & 0 & \ldots & 0 & -1 & \lambda_{L}
\end{array}\right) \text {, } \\
& i \hat{p}_{I}=\frac{1}{4}\left(\hat{p}_{F}-\hat{p}_{F}^{\dagger}+\hat{p}_{B}-\hat{p}_{B}^{\dagger}\right) \\
& =\frac{i}{2 a} \operatorname{diag}(1,0,0, \ldots, 0,0,-1) \text {. }
\end{aligned}
$$

The resulting momentum operator $\hat{p}_{R}+i \hat{p}_{I}$ is not Hermitian, but has a Hermitian component $\hat{p}_{R}$ and an anti-Hermitian component $i \hat{p}_{I}$, which is diagonal in the position basis. The Hermitian component $\hat{p}_{R}$ corresponds to a symmetrized forward-backward next-to-nearest neighbor derivative that extends over two lattice spacings.

The lattice eigenvalue problem of $\hat{p}_{R}$ is given by

$$
\hat{p}_{R} \phi_{k, x}=\frac{1}{a} \sin (k a) \phi_{k, x},
$$

and the momentum quantization condition takes the form

$$
\exp (2 i k L)=\frac{[1-\lambda \exp (i k a)]\left[1+\lambda_{L} \exp (i k a)\right]}{[\exp (i k a)+\lambda]\left[\exp (i k a)-\lambda_{L}\right]} .
$$

This relation reduces to Eq. (61) in the continuum limit. 
[1] A. L. Gaunt, T. F. Schmidutz, I. Gotlibovych, R. P. Smith, and Z. Hadzibabic, Bose-Einstein Condensation of Atoms in a Uniform Potential, Phys. Rev. Lett. 110, 200406 (2013).

[2] P. Harrison, Quantum Wells, Wires, and Dots (John Wiley and Sons, Ltd., Hoboken, New Jersey, 2005).

[3] A. Chodos, R. L. Jaffe, K. Johnson, C. B. Thorn, and V. F. Weisskopf, New extended model of hadrons, Phys. Rev. D 9 , 3471 (1974).

[4] A. Chodos, R. L. Jaffe, K. Johnson, and C. B. Thorn, Baryon structure in the bag theory, Phys. Rev. D 10, 2599 (1974).

[5] P. Hasenfratz and J. Kuti, The quark bag model, Phys. Rep. 40, 75 (1978).

[6] D. B. Kaplan, A method for simulating chiral fermions on the lattice, Phys. Lett. B 288, 342 (1992).

[7] Y. Shamir, Chiral fermions from lattice boundaries, Nucl. Phys. B 406, 90 (1993).

[8] G. Bonneau, J. Faraut, and G. Valent, Self-adjoint extensions of operators and the teaching of quantum mechanics, Am. J. Phys. 69, 322 (2001).

[9] M. H. Al-Hashimi and U.-J. Wiese, A new concept for the momentum of a quantum mechanical particle in a box, arXiv:2012.09596.

[10] H. B. Nielsen and M. Ninomiya, No-go theorem for regularizing chiral fermions, Phys. Lett. B 105, 219 (1981).

[11] H. B. Nielsen and M. Ninomiya, Absence of neutrinos on a lattice: (I). Proof by homotopy theory, Nucl. Phys. B 185, 20 (1981).

[12] D. Friedan, A proof of the Nielsen-Ninomiya theorem, Commun. Math. Phys. 85, 481 (1982).

[13] E. B. Davies and J. T. Lewis, An operational approach to quantum probability, Commun. Math. Phys. 17, 239 (1970).

[14] K. Kraus, General state changes in quantum theory, Ann. Phys. 64, 311 (1971).

[15] A. S. Holevo, Estimation of shift parameters of a quantum state, Rep. Math. Phys. 13, 379 (1978).

[16] A. S. Holevo, Covariant measurements and uncertainty relations, Rep. Math. Phys. 16, 385 (1979).

[17] E. B. Davies, Quantum Dynamics of Open Systems (Academic Press, New York, 1976).

[18] A. S. Holevo, Statistical Structure of Quantum Theory, Lecture Notes in Physics, Vol. 61 (Springer, Berlin, 2001).

[19] K. Kraus, States, Effects, and Operations: Fundamental Notions of Quantum Theory (Springer, Berlin, 1983).

[20] A. Peres, Neumark's theorem and quantum inseparability, Found. Phys. 20, 1441 (1990).

[21] M. Neumark, Spectral functions of a symmetric operator, Izv. Akad. Nauk USSR, Ser. Mat. 4, 277 (1940).

[22] M. Neumark, On a representation of additive operator set functions, C. R. Acad. Sci. URSS 41, 359 (1943).

[23] N. I. Akhiezer and I. M. Glazman, Theory of Linear Operators in Hilbert Space (Ungar, New York, 1963).

[24] C. W. Helstrom, Cramer-Rao inequalities for operator-valued measures in quantum mechanics, Int. J. Theor. Phys. 8, 361 (1973).
[25] F. Belgiorno and F. Gallone, Confined quantum systems and their limits, J. Math. Phys. 50, 022101 (2009).

[26] Y. Shikano and A. Hosoya, Optimal covariant measurement of momentum on a half line in quantum mechanics, J. Math. Phys. 49, 052104 (2008)

[27] J. von Neumann, Mathematische Grundlagen der Quantenmechanik (Springer, Berlin, 1932).

[28] M. Reed and B. Simon, Methods of Modern Mathematical Physics II, Fourier Analysis, Self-Adjointness (Academic Press, Inc., New York, 1975).

[29] F. Gieres, Mathematical surprises and Dirac's formalism in quantum mechanics, Rep. Prog. Phys. 63, 1893 (2000).

[30] T. Jurić, Observables in quantum mechanics and the importance of self-adjointness, arXiv:2103.01080.

[31] R. Balian and C. Bloch, Distribution of eigenfrequencies for the wave equation in a finite domain: I. Three-dimensional problem with smooth boundary surface, Ann. Phys. 60, 401 (1970).

[32] T. E. Clark, R. Menikoff, and D. H. Sharp, Quantum mechanics on the half-line using path integrals, Phys. Rev. D 22, 3012 (1980).

[33] E. Farhi and S. Gutmann, The functional integral on the halfline, Int. J. Mod. Phys. A 5, 3029 (1990).

[34] M. Carreau, E. Farhi, and S. Gutmann, Functional integral for a free particle in a box, Phys. Rev. D 42, 1194 (1990).

[35] M. H. Al-Hashimi and U.-J. Wiese, From a particle in a box to the uncertainty relation in a quantum dot and to reflecting walls for relativistic fermions, Ann. Phys. 327, 1 (2012).

[36] J. Twamley and G. J. Milburn, The quantum Mellin transform, New J. Phys. 8, 328 (2006).

[37] J. R. Klauder, Enhanced quantization: A primer, J. Phys. A: Math. Theor. 45, 285304 (2012).

[38] H. Bergeron and J.-P. Gazeau, Integral quantizations with two basic examples, Ann. Phys. 344, 43 (2014).

[39] C. R. Almeida, H. Bergeron, J.-P. Gazeau, and A. C. Scardua, Three examples of quantum dynamics on the half-line with smooth bouncing, Ann. Phys. 392, 206 (2018).

[40] L. Gouba, Affine quantization on the half line, J. High Energy Phys., Gravitation Cosmol. 7, 352 (2021).

[41] C. Cohen-Tannoudji, B. Dui, and F. Laloë, Quantum Mechanics I (Wiley, New York, 1977).

[42] P. Garbaczewski and W. Karwowski, Impenetrable barriers and canonical quantization, Am. J. Phys. 72, 924 (2004).

[43] M. H. Stone, Linear transformations in Hilbert space. III. Operational methods and group theory, Proc. Natl. Acad. Sci. USA 16, 172 (1930).

[44] J. von Neumann, Die Eindeutigkeit der Schrödingerschen Operatoren, Mathematische Annalen (Berlin) 104, 570 (1931).

[45] J. von Neumann, Über einen Satz von Herrn M. H. Stone, Ann. Math. 33, 567 (1932).

[46] M. H. Stone, On one-parameter unitary groups in Hilbert space, Ann. Math. 33, 643 (1932).

[47] H. Everett, Relative state formulation of quantum mechanics, Rev. Mod. Phys. 29, 454 (1957).

[48] F. Di Pumpo and M. Freyberger, Pointer-based model for state reduction in momentum space, Eur. Phys. J. D 73, 163 (2019). 